\title{
ON L-SPACES AND LEFT-ORDERABLE FUNDAMENTAL GROUPS
}

\author{
STEVEN BOYER, CAMERON MCA. GORDON, AND LIAM WATSON
}

\begin{abstract}
Examples suggest that there is a correspondence between L-spaces and 3manifolds whose fundamental groups cannot be left-ordered. In this paper we establish the equivalence of these conditions for several large classes of such manifolds. In particular, we prove that they are equivalent for any closed, connected, orientable, geometric 3-manifold that is non-hyperbolic, a family which includes all closed, connected, orientable Seifert fibred spaces. We also show that they are equivalent for the 2 -fold branched covers of non-split alternating links. To do this we prove that the fundamental group of the 2 -fold branched cover of an alternating link is left-orderable if and only if it is a trivial link with two or more components. We also show that this places strong restrictions on the representations of the fundamental group of an alternating knot complement with values in $\mathrm{HomeO}_{+}\left(S^{1}\right)$.
\end{abstract}

\section{INTRODUCTION}

In this paper all 3-manifolds will be assumed to be orientable.

Heegaard Floer homology is a package of 3-manifold invariants introduced by Ozsváth and Szabó [32, 33]. There are various versions of this theory, however for our purposes it will suffice to consider the simplest of these: the hat version, denoted $\widehat{\mathrm{HF}}$.

Definition 1. A closed, connected 3-manifold $Y$ is an L-space if it is a rational homology sphere with the property that $\mathrm{rk} \widehat{\mathrm{HF}}(Y)=\left|H_{1}(Y ; \mathbb{Z})\right|$.

L-spaces form the class of manifolds with minimal Heegaard Floer homology and are of interest for various reasons. For instance, such manifolds do not admit co-orientable taut foliations [31, Theorem 1.4]. It is natural to ask if there are characterizations of L-spaces which do not reference Heegaard Floer homology (cf. [35, Question 11]). Examples of Lspaces include lens spaces as well as all connected sums of manifolds with elliptic geometry [36. Proposition 2.3]. These examples also enjoy the property that their fundamental groups cannot be left-ordered:

Definition 2. A non-trivial group $G$ is called left-orderable if there exists a strict total ordering $<$ on its elements such that $g<h$ implies $f g<f h$ for all elements $f, g, h \in G$.

Date: July 25, 2011.

Steven Boyer was partially supported by NSERC grant RGPIN 9446-2008.

Cameron Gordon was partially supported by NSF grant DMS-0906276.

Liam Watson was partially supported by an NSERC postdoctoral fellowship. 
While the trivial group obviously satisfies this criterion, in this paper we will adopt the convention that it is not left-orderable.

The left-orderability of 3-manifold groups has been studied in work of Boyer, Rolfsen and Wiest [3. An argument of Howie and Short [18, Lemma 2] shows that the fundamental group of an irreducible 3-manifold $M$ with positive first Betti number is locally indicable, hence left-orderable [4]. More generally, such a group is left-orderable if it admits an epimorphism to a left-orderable group [3, Theorem 1.1(1)].

The aim of this note is to establish a connection between L-spaces and the left-orderability of their fundamental groups. Given the results we obtain and those obtained elsewhere [6, 7, 8, 40], we formalise a question which has received attention in the recent literature in the following conjecture.

Conjecture 3. An irreducible rational homology 3-sphere is an L-space if and only if its fundamental group is not left-orderable.

It has been asked by Ozsváth and Szabó whether L-spaces can be characterized as those closed, connected 3-manifolds admitting no co-orientable taut foliations. Thus, in the context of Conjecture 3 it is interesting to consider the following open questions: Does the existence of a co-orientable taut foliation on an irreducible rational homology 3 -sphere imply the manifold has a left-orderable fundamental group? Are the two conditions equivalent? Calegari and Dunfield have shown that the existence of a co-orientable taut foliation on an irreducible atoroidal rational homology 3 -sphere $Y$ implies that $\pi_{1}(Y)$ has a left-orderable finite index subgroup [5, Corollary 7.6]. Of course, an affirmative answer to Conjecture 3 , combined with [31, Theorem 1.4], would prove that the existence of a co-orientable taut foliation implies left-orderable fundamental group.

Our first result verifies the conjecture in the case of Seifert fibred spaces.

Theorem 4. Suppose $Y$ is a closed, connected, Seifert fibred 3-manifold. Then $Y$ is an $L$-space if and only if $\pi_{1}(Y)$ is not left-orderable.

The proof of this theorem in the case where the base orbifold of $Y$ is orientable depends on results of Boyer, Rolfsen and Wiest [3] and Lisca and Stipsicz [26]. This case of Theorem [4 has been independently observed by Peters [40. There are, on the other hand, many Seifert fibred rational homology 3-spheres with non-orientable base orbifolds, and it is shown in [3] that such manifolds have non-left-orderable fundamental groups. Theorem 4 therefore yields an interesting class of L-spaces that, to the best of our knowledge, has not received attention in the literature (see also [48, Theorem 3.32]).

The set of torus semi-bundles provides another interesting family of 3-manifolds in which to examine the relationship between L-spaces and non-left-orderable fundamental groups. Such manifolds are unions of two twisted $I$-bundles over the Klein bottle and are either Seifert fibred or admit a Sol geometry. Indeed, let $N$ be a twisted $I$-bundle over the Klein bottle and set $T=\partial N$. There are distinguished slopes $\phi_{0}, \phi_{1}$ on $T$ corresponding to the two Seifert structures supported by $N$. Here $\phi_{0}$ denotes the fibre slope of the structure with 
base orbifold a Möbius band and $\phi_{1}$ that with base orbifold $D^{2}(2,2)$. The general torus semi-bundle is homeomorphic to an identification space $W(f)=N \cup_{f} N$ where $f: T \rightarrow T$ is a homeomorphism. Further, $W(f)$ is

- a Seifert fibre space if and only if $f$ identifies $\phi_{i}$ with $\phi_{j}$ for some $i, j \in\{0,1\}$.

- a Sol manifold if and only if $f$ does not identify any $\phi_{i}$ with any $\phi_{j}$ for $i, j \in\{0,1\}$.

- a rational homology 3 -sphere if and only if $f$ does not identify $\phi_{0}$ with $\phi_{0}$.

Thus the generic torus semi-bundle is a rational homology sphere and a Sol manifold.

Theorem 5. Suppose that $W$ is a torus semi-bundle. Then the following statements are equivalent:

(a) $H_{1}(W ; \mathbb{Q}) \cong 0$.

(b) $\pi_{1}(W)$ is not left-orderable.

(c) $W$ is an L-space.

A key step in the proof of this result requires a computation of the bordered Heegaard Floer homology [25] of the twisted $I$-bundle over the Klein bottle. An immediate consequence of it verifies Conjecture 3 for Sol manifolds.

Corollary 6. Suppose that $Y$ is a closed, connected 3-manifold with Sol geometry. Then $Y$ is an L-space if and only if $\pi_{1}(Y)$ is not left-orderable.

Theorem 4 and Corollary 6 combine to give the following general statement:

Theorem 7. Suppose that $Y$ is a closed, connected, geometric, non-hyperbolic 3-manifold. Then $Y$ is an L-space if and only if $\pi_{1}(Y)$ is not left-orderable.

Ozsváth and Szabó determined a large family of L-spaces - the 2-fold covers of $S^{3}$ branched over a non-split alternating link [37, Proposition 3.3]. Conjecture 3 can be established in this setting as well. We prove:

Theorem 8. The fundamental group of the 2-fold branched cover of an alternating link $L$ is left-orderable if and only if $L$ is a trivial link with two or more components. In particular, the fundamental group of the 2-fold branched cover of a non-split alternating link is not left-orderable.

Note that generically, the 2 -fold branched cover of an alternating link $L$ is hyperbolic.

Josh Greene [14 has found an alternate proof of Theorem 8. There is also relevant recent work of Ito on 2-fold branched covers [19] and Levine and Lewallen on strong L-spaces (manifolds $Y$ for which $\widehat{\mathrm{CF}}(Y) \cong \widehat{\mathrm{HF}}(Y))$ [23].

The results above relate L-spaces and manifolds with non-left-orderable fundamental groups. Next we consider examples of non-L-spaces with left-orderable fundamental groups. An 
interesting family of non-L-spaces has been constructed by Ozsváth and Szabó - those manifolds obtained by non-trivial surgery on a hyperbolic alternating knot [36. Theorem $1.5]$.

Recall that a special alternating knot is a knot which has an alternating diagram each of whose Seifert circles bounds a complementary region of the diagram. Equivalently, it is an alternating knot such that either each of the crossings in a reduced diagram for the knot is positive or each is negative.

Proposition 9. Let $K$ be a prime alternating knot in $S^{3}$.

(1) If $q \neq 0$ and $S_{p / q}^{3}(K)$ is Seifert fibred, then $\pi_{1}\left(S_{p / q}^{3}(K)\right)$ is left-orderable.

(2) If $K$ is not a special alternating knot, then $\pi_{1}\left(S_{1 / q}^{3}(K)\right)$ is left-orderable for all non-zero integers $q$.

(3) If $K$ is a special alternating knot, then either all crossings in a reduced diagram for $K$ are positive and $\pi_{1}\left(S_{1 / q}^{3}(K)\right)$ is left-orderable for all positive integers $q$, or all crossings in the diagram are negative and $\pi_{1}\left(S_{1 / q}^{3}(K)\right)$ is left-orderable for all negative integers $q$.

In the case of the figure eight knot we can say a little more.

Proposition 10. Let $K$ be the figure eight knot. If $-4<r=\frac{p}{q}<4$, then $\pi_{1}\left(S_{r}^{3}(K)\right)$ is left-orderable.

Clay, Lidman and Watson have shown that the fundamental group of \pm 4 -surgery on the figure eight knot is left-orderable [6, §4].

It is evident that a non-trivial subgroup of a left-orderable group is left-orderable. Here is a question which arises naturally from the ideas of this paper.

Question 11. Is a rational homology 3-sphere finitely covered by an L-space necessarily an L-space?

We point out that it follows from Theorem 5 that there exists a class of examples of 2 -fold covers that behave in this way: each $\mathbb{Q}$-homology sphere with Sol geometry admits a 2-fold cover which is a $\mathbb{Q}$-homology sphere with Sol geometry.

The non-left-orderability of the fundamental group of the 2-fold branched cover of a prime knot in the 3-sphere has an interesting consequence for certain representations of the fundamental group of its complement.

Theorem 12. Let $K$ be a prime knot in the 3-sphere and suppose that the fundamental group of its 2-fold branched cyclic cover is not left-orderable. If $\rho: \pi_{1}\left(S^{3} \backslash K\right) \rightarrow \mathrm{Homeo}_{+}\left(S^{1}\right)$ is a homomorphism such that $\rho\left(\mu^{2}\right)=1$ for some meridional class $\mu$ in $\pi_{1}\left(S^{3} \backslash K\right)$, then the image of $\rho$ is either trivial or isomorphic to $\mathbb{Z} / 2$. 
Corollary 13. Let $K$ be an alternating knot and $\rho: \pi_{1}\left(S^{3} \backslash K\right) \rightarrow$ Homeo $_{+}\left(S^{1}\right)$ a homomorphism. If $\rho\left(\mu^{2}\right)=1$ for some meridional class $\mu$ in $\pi_{1}\left(S^{3} \backslash K\right)$, then the image of $\rho$ is either trivial or isomorphic to $\mathbb{Z} / 2$.

Alan Reid has pointed out the following consequence of this corollary.

Corollary 14. Suppose that $K$ is an alternating knot and let $\mathcal{O}_{K}(2)$ denote the orbifold with underlying set $S^{3}$ and singular set $K$ with cone angle $\pi$. Suppose further that $\mathcal{O}_{K}(2)$ is hyperbolic. If the trace field of $\pi_{1}\left(\mathcal{O}_{K}(2)\right)$ has a real embedding, then it must determine a PSU(2)-representation. In other words, the quaternion algebra associated to $\pi_{1}\left(\mathcal{O}_{K}(2)\right)$ is ramified at that embedding.

Outline. The paper is organized as follows. Theorem 4 is proven in $\oint 2$. Generalities on torus semi-bundles are dealt with in 93 followed by an outline of an inductive proof of Theorem 5. The base case of the induction is dealt with in 84 and the inductive step in 95 . Theorem 8 is proven in 86 while Propositions 9 and 10 are dealt with in 97 . Finally, in 88 we prove Theorem 12 and Corollaries 13 and 14 .

Acknowledgements. The authors thank Adam Clay, Josh Greene, Tye Lidman and Ciprian Manolescu for their comments on and interest in this work, Alan Reid for mentioning Corollary 14, Michael Polyak for showing them the presentation described in Section 3.1, and Józef Przytycki for pointing out Wada's paper [46]. They also thank Adam Levine, Robert Lipshitz, Peter Ozsváth and Dylan Thurston for patiently answering questions about bordered Heegaard Floer homology, which proved to be a key tool for establishing Corollary 6.

\section{A characterization of Seifert fibred L-SPaCes}

2.1. Preliminaries on L-spaces. We recall an important construction which gives rise to infinite families of L-spaces (see [36, Section 2]).

Definition 15. Let $M$ be a compact, connected 3-manifold with torus boundary. Given a basis $\{\alpha, \beta\} \subset H_{1}(\partial M)$ the triple $(\alpha, \beta, \alpha+\beta)$ will be referred to as a triad whenever

$$
\left|H_{1}(M(\alpha+\beta) ; \mathbb{Z})\right|=\left|H_{1}(M(\alpha) ; \mathbb{Z})\right|+\left|H_{1}(M(\beta) ; \mathbb{Z})\right| .
$$

Note that our boundary orientation differs from that of Ozsváth and Szabó, resulting in a sign discrepancy in the definition of a triad.

Proposition 16 (Proposition 2.1 of [36]). If $M$ admits a triad $(\alpha, \beta, \alpha+\beta)$ with the property that $M(\alpha)$ and $M(\beta)$ are L-spaces, then $M(\alpha+\beta)$ is an L-space as well.

It follows by induction that each of the manifolds $M(n \alpha+\beta)$ is an L-space for $n \geq 0$. More generally, we include a short proof of the following well-known fact: 
Proposition 17 (See Example 1.10 of [38]). Suppose that $M$ admits a triad $(\alpha, \beta, \alpha+\beta)$ with the property that $M(\alpha)$ and $M(\beta)$ are L-spaces. Then for all coprime pairs $p, q \geq 0$, $M(p \alpha+q \beta)$ is an L-space.

Proof. Let $p, q$ be a coprime pair with $p, q \geq 0$. Without loss of generality we can suppose $p, q \geq 1$. Choose integers $a_{1} \geq 0$ and $a_{2}, \ldots, a_{r} \geq 1$ such that

$$
\frac{p}{q}=\left[a_{1}, a_{2}, \ldots, a_{r}\right]=a_{1}+\frac{1}{a_{2}+\cdots \frac{1}{a_{r}}}
$$

Let $r\left(\frac{p}{q}\right) \geq 1$ be the minimal $r$ such that $\frac{p}{q}$ admits such an expansion. When $r\left(\frac{p}{q}\right)=1$, the proposition follows from the remark after Proposition 16

Assume next that $r=r\left(\frac{p}{q}\right) \geq 2$ and that $M\left(p^{\prime} \alpha+q^{\prime} \beta\right)$ is an L-space for all coprime pairs $p^{\prime}, q^{\prime} \geq 1$ such that $r\left(\frac{p^{\prime}}{q^{\prime}}\right)<r$. Write $\frac{p}{q}=\left[a_{1}, a_{2}, \ldots, a_{r}\right]$ as above and note that as $\left[a_{1}, a_{2}, \ldots, a_{r-1}, 1\right]=\left[a_{1}, a_{2}, \ldots, a_{r-1}+1\right]$ we have $a_{r} \geq 2$. Set

$$
\frac{p_{1}}{q_{1}}=\left[a_{1}, a_{2}, \ldots, a_{r-1}\right]
$$

and

$$
\frac{p_{2}}{q_{2}}=\left[a_{1}, a_{2}, \ldots, a_{r}-1\right]
$$

It follows from the basic properties of the convergents of a continued fraction that $\left\{p_{1} \alpha+\right.$ $\left.q_{1} \beta, p_{2} \alpha+q_{2} \beta\right\}$ is a basis of $H_{1}(\partial M)$ and $\frac{p}{q}=\frac{p_{1}+p_{2}}{q_{1}+q_{2}}$. The latter shows that

$$
\left|H_{1}(M(p \alpha+q \beta) ; \mathbb{Z})\right|=\left|H_{1}\left(M\left(p_{1} \alpha+q_{1} \beta\right) ; \mathbb{Z}\right)\right|+\left|H_{1}\left(M\left(p_{2} \alpha+q_{2} \beta\right) ; \mathbb{Z}\right)\right| .
$$

(See the proof of [47, Theorem 4.7].) This establishes that $\left(p_{1} \alpha+q_{1} \beta, p_{2} \alpha+q_{2} \beta, p \alpha+q \beta\right)$ is a triad. Our induction hypothesis implies that $M\left(p_{1} \alpha+q_{1} \beta\right)$ is an L-space. We also have that $M(p \alpha+q \beta)$ is an L-space as long as $M\left(p_{2} \alpha+q_{2} \beta\right)$ is one. The latter will be true if $r\left(\frac{p_{2}}{q_{2}}\right)<r$, but this may not be the case. On the other hand if $r\left(\frac{p_{2}}{q_{2}}\right)=r$, we must have $a_{r}-1 \geq 2$. Thus a second induction on $a_{r}$ is sufficient to complete the proof.

For example, all sufficiently large surgeries on a Berge knot (the conjecturally complete list of knots in $S^{3}$ admitting lens space surgeries [1]) yield L-spaces.

2.2. Seifert fibred L-spaces. Our notation for Seifert fibred spaces follows that of Boyer, Rolfsen and Wiest 3 and is consistent with that of Scott [44. Let $Y$ be Seifert fibred with base orbifold $\mathcal{B}$, and write $\mathcal{B}=B\left(a_{1}, a_{2}, \ldots, a_{n}\right)$ for some surface $B$ with cone points of order $a_{i}>1$. If $Y$ is a rational homology sphere then $B$ is either $S^{2}$ or $P^{2}$.

Lisca and Stipsicz have shown [26, Theorem 1.1] that when $B=S^{2}, Y$ is an L-space if and only if $Y$ does not admit a horizontal foliation while Boyer, Rolfsen and Wiest proved that these $Y$ do not admit a horizontal foliation if and only if $\pi_{1}(Y)$ is not left-orderable [3, Theorem 1.3(b)]. Thus Theorem 4 holds when $B=S^{2}$. (See also Peters [40].) To complete its proof, we must consider the case $B=P^{2}$. 
2.3. The proof of Theorem 4 when $B=P^{2}$. Let $Y$ be Seifert fibred with base orbifold $\mathcal{B}=P^{2}\left(a_{1}, a_{2}, \ldots, a_{n}\right)$. By [3], $\pi_{1}(Y)$ is not left-orderable. Since $Y$ is orientable but $P^{2}$ is not, $Y$ is a rational homology sphere. Hence we are reduced to establishing the following proposition:

Proposition 18. Suppose $Y$ is a Seifert fibred space with base orbifold $\mathcal{B}=P^{2}\left(a_{1}, a_{2}, \ldots, a_{n}\right)$ where $a_{i} \geq 1$ if $n=1$ and $a_{i} \geq 2$ otherwise. Then $Y$ is an $L$-space.

Proof. First suppose that $\mathcal{B}=P^{2}\left(a_{1}\right)$ where $a_{1} \geq 1$. Then $Y$ is obtained by filling $N=$ $K \tilde{\times} I$, the twisted $I$-bundle over the Klein bottle, along some slope $\alpha$. The Seifert structure on $Y$ restricts to a circle bundle structure on $N$ with base space the Möbius band. If $\phi_{0}$ is the fibre of this bundle, then $N\left(\phi_{0}\right) \cong S^{1} \times S^{2}$. Further, $\Delta\left(\alpha, \phi_{0}\right)=a_{1}$ by Heil [17].

There is another Seifert structure on $N$ with base orbifold $D^{2}(2,2)$ and fibre $\phi_{1}$ such that $\Delta\left(\phi_{1}, \phi_{0}\right)=1$. Then $Y=N(\alpha)$ is either the L-space $N\left(\phi_{1}\right) \cong P^{3} \# P^{3}$ or it admits a Seifert structure over $S^{2}\left(2,2, \Delta\left(\alpha, \phi_{1}\right)\right)$ where $\Delta\left(\alpha, \phi_{1}\right) \geq 1$. (See [17].) Since $\alpha \neq \phi_{0}, Y$ is elliptic in the latter case and therefore is also an L-space [36, Proposition 2.3]. Thus the proposition holds when $Y$ has at most one exceptional fibre.

Suppose inductively that any Seifert fibred manifold with base orbifold $P^{2}\left(a_{1}, \ldots, a_{r}\right)$ is an L-space whenever $1 \leq r \leq n$. Fix a Seifert fibred manifold over $P^{2}\left(a_{1}, \ldots, a_{n+1}\right)$ and recall that by hypothesis, $a_{i} \geq 2$ for all $i$.

Let $\phi_{0}$ be the exceptional fibre of $Y$ corresponding to the cone point of index $a_{n+1}$ and denote the exterior of $\phi_{0}$ in $Y$ by $N$. Then $N$ is a Seifert fibred manifold with base orbifold $B_{0}\left(a_{1}, a_{2}, \ldots, a_{n}\right)$ where $B_{0}$ is a Möbius band. The rational longitude of $N$ is the unique slope $\phi_{0}$ on $\partial N$ which represents a torsion element of $H_{1}(N ; \mathbb{Z})$. Since $N\left(\phi_{0}\right) \cong S^{1} \times S^{2}$, $\lambda_{N}=\phi_{0}$

It is convenient to identify the (oriented) slopes on $\partial N$ with primitive elements of $H_{1}(\partial N ; \mathbb{Z})$. Choosing a dual class $\mu \in H_{1}(\partial N ; \mathbb{Z})$ for $\phi_{0}$ (i.e. a class such that $\mu \cdot \phi_{0}=1$ ), we obtain a basis for $H_{1}(\partial N ; \mathbb{Z})$. For any slope $\gamma \neq \pm \phi_{0}$, we can write $\gamma= \pm\left(p \mu+q \phi_{0}\right)$ where $p \geq 1$. The Dehn filling $N(\gamma)$ is Seifert fibred with base orbifold $P^{2}\left(a_{1}, \ldots, a_{n}, \Delta\left(\phi_{0}, \gamma\right)\right)=$ $P^{2}\left(a_{1}, \ldots, a_{n}, p\right)$. In particular, if $\alpha$ denotes the meridional slope of $\phi_{0}$, then $Y=N(\alpha)$ so that $\alpha= \pm\left(a_{n+1} \mu+q \phi_{0}\right)$. Note as well that for any $q \in \mathbb{Z}$, our induction hypothesis implies that $N\left(\mu+q \phi_{0}\right)$ is an L-space.

By [47, Lemma 2.1], there is a constant $D_{N}>0$ depending only on $N$ such that for each slope $\gamma$ on $\partial N,\left|H_{1}(N(\gamma) ; \mathbb{Z})\right|=D_{N} \Delta\left(\gamma, \phi_{0}\right)$. Then as $\mu \cdot \phi_{0}=1$,

$$
\begin{aligned}
\left|H_{1}\left(N\left(2 \mu+\phi_{0}\right) ; \mathbb{Z}\right)\right| & =D_{N} \Delta\left(2 \mu+\phi_{0}, \phi_{0}\right) \\
& =D_{N} \Delta\left(\mu, \phi_{0}\right)+D_{N} \Delta\left(\mu+\phi_{0}, \phi_{0}\right) \\
& =\left|H_{1}(N(\mu) ; \mathbb{Z})\right|+\left|H_{1}\left(N\left(\mu+\phi_{0}\right) ; \mathbb{Z}\right)\right| .
\end{aligned}
$$

It follows that $\left(\mu, \mu+\phi_{0}, 2 \mu+\phi_{0}\right)$ is a triad of slopes on $\partial N$. Since $N(\mu)$ and $N\left(\mu+\phi_{0}\right)$ are L-spaces, Proposition 17 implies that $N\left((p+q) \mu+q \phi_{0}\right)$ is an L-space for all coprime pairs $p, q \geq 0$. Now $\left\{\frac{p+q}{q}: p, q \geq 0\right.$ are coprime $\}=(\mathbb{Q} \cap[1, \infty)) \cup\left\{\frac{1}{0}\right\}$ so that $N(\gamma)$ is an 
L-space for all slopes $\gamma$ in the sector of $H_{1}(\partial N ; \mathbb{R})$ bounded by the lines $\left\{t \mu: t \in \mathbb{R}_{+}\right\}$and $\left\{t\left(\mu+\phi_{0}\right): t \in \mathbb{R}_{+}\right\}$. Since $\mu$ was chosen as an arbitrary dual class to $\phi_{0}$, given an integer $q, N(\gamma)$ is an L-space for all $\gamma \in \mathcal{S}_{q}$ where $\mathcal{S}_{q}$ is the set of slopes in the sector of $H_{1}(\partial N ; \mathbb{R})$

bounded by the lines $\left\{t\left(\mu+q \phi_{0}\right): t \in \mathbb{R}_{+}\right\}$and $\left\{t\left(\mu+(q+1) \phi_{0}\right): t \in \mathbb{R}_{+}\right\}$. Then as $\bigcup_{q=-\infty}^{\infty} \mathcal{S}_{q}$ is the set of slopes on $\partial N$ other than $\phi_{0}$, the proposition has been proved.

\section{TORUS SEMI-BUNDLES}

Let $N$ be an oriented twisted $I$-bundle over the Klein bottle and give $T=\partial N$ the induced orientation. We remarked in the proof of Proposition 18 that there are two distinguished slopes $\phi_{0}, \phi_{1}$ on $T$ corresponding to the two Seifert structures supported by $N$. Here $\phi_{0}$ is the fibre slope of the structure with base orbifold a Möbius band while $\phi_{1}$ is the slope of the structure with base orbifold $D^{2}(2,2)$. It is well-known that $\phi_{0}$ and $\phi_{1}$ can be oriented so that $\phi_{0} \cdot \phi_{1}=1$. Do this and observe that $\left\{\phi_{0}, \phi_{1}\right\}$ is a basis for $H_{1}(T)$. We will identify the mapping class group of $T$ with $G L_{2}(\mathbb{Z})$ using this basis - the mapping class of a homeomorphism $f$ corresponds to the matrix of $f_{*}: H_{1}(T ; \mathbb{Z}) \rightarrow H_{1}(T ; \mathbb{Z})$ with respect to $\left\{\phi_{0}, \phi_{1}\right\}$.

The first homology of $T$ maps to a subgroup of index two in $H_{1}(N ; \mathbb{Z})$. In fact, $H_{1}(N ; \mathbb{Z}) \cong$ $\mathbb{Z} \oplus \mathbb{Z} / 2$ where $\phi_{0}$ generates the second factor and $\phi_{1}$ represents twice a generator of the first. It follows that $\phi_{0}$ is the rational longitude of $N$ and that for any slope $\gamma$ on $T$ we have

$$
\left|H_{1}(N(\gamma) ; \mathbb{Z})\right|=4 \Delta\left(\gamma, \phi_{0}\right)
$$

Further, it is well-known that a filling of $N$ with finite first homology is either $P^{3} \# P^{3}$ or admits an elliptic geometry. Hence $N(\gamma)$ is an L-space if and only if $\gamma \neq \phi_{0}$.

Let $f$ be a homeomorphism of $T=\partial N$ and suppose that $f_{*}=\left(\begin{array}{ll}a & b \\ c & d\end{array}\right)$ with respect to $\phi_{0}, \phi_{1}$. Then $W(f)=N \cup_{f}\left(-\operatorname{det}\left(f_{*}\right)\right) N$ is an oriented torus semi-bundle and each such semi-bundle can be obtained this way. We claim that

$$
\left|H_{1}(W(f) ; \mathbb{Z})\right|=16|c|
$$

In fact, if $M_{1}, M_{2}$ are two rational homology solid tori and $W=M_{1} \cup_{f} M_{2}$, it follows from the homology exact sequence of the pair $\left(W, M_{1}\right)$ that $\left|H_{1}(W ; \mathbb{Z})\right|=d_{1} d_{2}\left|T_{1}\right|\left|T_{2}\right| \Delta\left(\lambda_{1}, \lambda_{2}\right)$ where $\lambda_{j}$ is the rational longitude of $M_{j}, d_{j} \geq 1$ is its order in $H_{1}\left(M_{j} ; \mathbb{Z}\right)$, and $T_{j}$ is the torsion subgroup of $H_{1}\left(M_{j} ; \mathbb{Z}\right)$. In our case, $d_{1}=d_{2}=\left|T_{1}\right|=\left|T_{2}\right|=2$ and $\Delta\left(\lambda_{1}, \lambda_{2}\right)=$ $\Delta\left(\phi_{0}, f_{*}\left(\phi_{0}\right)\right)=|c|$. Thus $\left|H_{1}(W(f) ; \mathbb{Z})\right|=16|c|$ as claimed. It follows that a torus semibundle $W$ is a rational homology 3 -sphere if and only if $|c| \geq 1$.

Remark 19. The fibre classes $\phi_{0}, \phi_{1}$ are preserved up to sign by each homeomorphism of $N$. In fact, under the identification of the mapping class group of $T$ with $G L_{2}(\mathbb{Z})$ described above, the image of the mapping class group of $N$ in that of $T$ is the subgroup $\left\{ \pm\left(\begin{array}{ll}1 & 0 \\ 0 & 1\end{array}\right), \pm\left(\begin{array}{cc}1 & 0 \\ 0 & -1\end{array}\right)\right\}$. Hence given a torus semi-bundle $W(f)$, we can always assume $f_{*}=$ $\left(\begin{array}{ll}a & b \\ c & d\end{array}\right)$ where $c \geq 0$ and $\operatorname{det}\left(f_{*}\right)$ is an arbitrary element of $\{ \pm 1\}$. 
Proof of Theorem 5. The implication (c) $\Rightarrow$ (a) of the theorem is immediate. The implication $(\mathrm{a}) \Rightarrow(\mathrm{b})$ is a consequence of the proof of [3. Proposition 9.1(1)]. There it is shown that if a torus semi-bundle $W(f)$ has a left-orderable fundamental group, then $\phi_{0}$ must

be identified with $\pm \phi_{0}$ by $f_{*}$. Equivalently, $f_{*}=\left(\begin{array}{ll}a & b \\ 0 & d\end{array}\right)$ with respect to the basis $\phi_{0}, \phi_{1}$ of $H_{1}(T ; \mathbb{Z})$, and therefore $W(f)$ is not a rational homology 3 -sphere.

To complete the proof of Theorem 5, we must show that the implication (b) $\Rightarrow$ (c) holds. To that end, let $W(f)$ be a torus semi-bundle whose fundamental group is not left-orderable and suppose that $f_{*}=\left(\begin{array}{ll}a & b \\ c & d\end{array}\right)$ with respect to $\phi_{0}, \phi_{1}$. Note that $c \neq 0$ as otherwise $W(f)$ would be irreducible and have a positive first Betti number, so its fundamental group would be left-orderable [3, Theorem 1.1(1)]. Thus $|c| \geq 1$. By Remark 19 we can suppose $c \geq 1$ and $\operatorname{det}\left(f_{*}\right)=-1$. We will proceed by induction on $c$. The initial case $c=1$ is dealt with in the next section using a bordered Heegaard Floer homology argument. See Theorem 22. The inductive step is handled in $\$ 5$ using a surgery argument based on the the triad condition of Proposition 16. See Proposition 23.

\section{The Bordered invariants of the twisted $I$-Bundle over the Klein Bottle.}

Heegaard Floer homology has been extended to manifolds with connected boundary by Lipshitz, Ozsváth and Thurston [25] (this approach subsumes knot Floer homology [34, 42] and was preceded, for the case of sutured manifolds, by work of Juhász [20]). In this context, the invariants take the form of certain modules (described below) over a unital differential (graded) algebra $\mathcal{A}$. Denote by $\mathcal{I} \subset \mathcal{A}$ the subring of idempotents. Our focus is on the bordered invariants of the twisted $I$-bundle over the Klein bottle, and as such we restrict our attention to the case of manifolds with torus boundary. This simplifies some of the objects in question, and the relevant setup in this case is summarized nicely in the work of Levine [22]. As such we will adhere to the notation and conventions of [22, Section 2] in the arguments and calculations that follow. We work with $\mathbb{F}=\mathbb{Z} / 2$ coefficients throughout.

4.1. Determining the bordered invariants. Recall that a (left) type D structure over $\mathcal{A}$ is an $\mathbb{F}$-vector space $V$ equipped with a left action of $\mathcal{I}$ such that $V=\iota_{0} V \oplus \iota_{1} V$ and a map $\delta_{1}: V \rightarrow \mathcal{A} \otimes_{\mathcal{I}} V$ (satisfying a compatibility condition, see [22, Equation (3)], for example).

The twisted $I$-bundle over the Klein bottle $N$ is described by the bordered Heegaard diagram in Figure 1. Recall that $N$ may be constructed by identifying a pair of solid tori along an essential annulus, giving rise to the Seifert structure with base orbifold $D^{2}(2,2)$. This identification of solid tori is reflected in the calculation of $\pi_{1}(N)=\left\langle a, b \mid a^{2} b^{2}\right\rangle$ via Seifertvan Kampen; alternatively, this presentation for $\pi_{1}(N)$ may be obtained using the single $\alpha$-curve in Figure 1 to obtain the relation. The reader may verify that the framing specified by this bordered diagram is consistent with $\left\{\phi_{0}, \phi_{1}\right\}$ by, for example, noting that the words $a b$ and $b^{2}$ (read from the $\operatorname{arcs} \alpha_{2}^{a}$ and $\alpha_{1}^{a}$, respectively) correspond to the peripheral elements $\phi_{0}$ and $\phi_{1}$, respectively. 


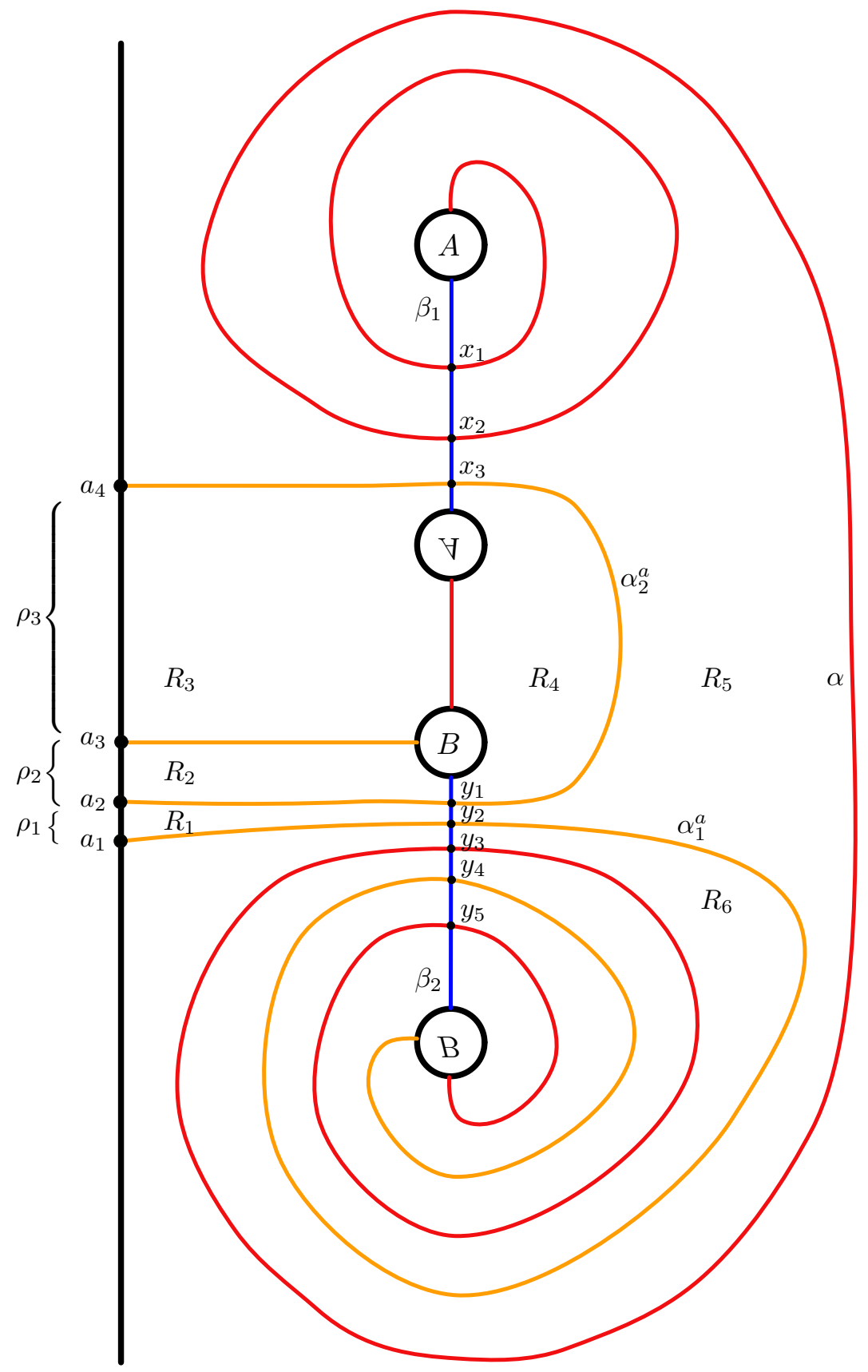

FiguRE 1. A genus 2 bordered Heegaard diagram for the twisted $I$-bundle over the Klein bottle. The circles marked $A$ and $B$ are identified to give the handles, and the pair of twists in each handle encodes the Seifert structure with base orbifold $D^{2}(2,2)$. 
Let $\mathbf{D}=\widehat{\mathrm{CFD}}(N)$. Our convention for the decomposition from the left-action of $\mathcal{I}$ is to denote by $\circ$ those generators $x$ for which $\iota_{0} x=x$, and by $\bullet$ those generators $y$ for which $\iota_{1} y=y$.

Proposition 20. The type D structure $\mathbf{D}$ is described by the directed graph
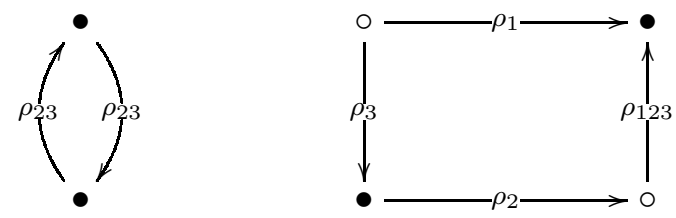

The requisite map $\delta_{1}: V \rightarrow \mathcal{A} \otimes_{\mathcal{I}} V$ for the type $\mathrm{D}$ structure is read from this directed graph as follows. The edge ${ }_{\circ}^{x}-\rho_{1} \rightarrow y$, for example, indicates that there is a generator $x$ for which $\iota_{0} x=x$ and a generator $y$ for which $\iota_{1} y=y$ such that $\rho_{1} \otimes y$ appears as a summand in the expression for $\delta_{1}(x)$. Since $\mathbf{D}$ decomposes into two summands, it will be convenient to write $\mathbf{D}=\mathbf{D}_{\mathbf{1}} \oplus \mathbf{D}_{\mathbf{2}}$ where the subscripts $\mathbf{1}$ an $\mathbf{2}$ denote the left and right connected components of the directed graph in Proposition 20, respectively.

Proof of Proposition 20. We determine $\mathbf{D}$ directly from the definition, using the bordered Heegaard digram in Figure 1. There are 8 generators for the underlying vector space $V$, partitioned according to

$$
\begin{aligned}
& \iota_{0} V=\left\langle\left(x_{1}, y_{1}\right),\left(x_{2}, y_{1}\right),\left(x_{3}, y_{3}\right),\left(x_{3}, y_{5}\right)\right\rangle \\
& \iota_{1} V=\left\langle\left(x_{1}, y_{2}\right),\left(x_{1}, y_{4}\right),\left(x_{2}, y_{2}\right),\left(x_{2}, y_{4}\right)\right\rangle
\end{aligned}
$$

Adhering to the conventions for the case of a torus boundary outlined in [22, Section 2], we begin by listing the possible domains in Table 1 . This follows from a case study, having observed that there are only 4 general types of domains $D$ depending on $n_{1} R_{3}+n_{2} R_{4} \subseteq D$ where the only possible multiplicities are

$$
\left(n_{1}, n_{2}\right) \in\{(0,0),(0,1),(1,1),(1,2)\}
$$

(see [22, Equation (4)], for example).

The contributions of $D_{1}$ and $D_{2}$ to $\delta_{1}$ are immediate, as these regions are a bigon (containing the Reeb chord $-\rho_{1}$ ) and a rectangle (containing the Reeb chord $-\rho_{2}$ ), respectively. As a result we have operations

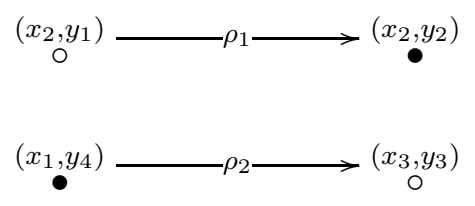

in $\mathbf{D}$ (specifically, $\mathbf{D}_{\mathbf{2}}$ ). Notice that potential contributions from the domains $R_{2}+R_{3}+$ $R_{4}+R_{5}+R_{6}$ and $R_{2}+R_{3}+2 R_{4}$ (each of which would contribute a $\rho_{23}$ ) are ruled out since they are inconsistent with the idempotents: $\left(x_{2}, y_{1}\right),\left(x_{3}, y_{3}\right) \in \iota_{0} \mathbf{D}_{\mathbf{2}}$. 


\begin{tabular}{|l|l|l|l|}
\hline Domain & Source & Target & Notes and labels \\
\hline \hline$R_{1}$ & $\left(x_{1}, y_{1}\right)$ & $\left(x_{1}, y_{2}\right)$ & Lost in cancelation. \\
& $\left(x_{2}, y_{1}\right)$ & $\left(x_{2}, y_{2}\right)$ & $D_{1}$ \\
\hline \hline$R_{4}$ & $\left(x_{1}, y_{1}\right)$ & $\left(x_{3}, y_{5}\right)$ & This is the only provincial domain. \\
\hline$R_{2}+R_{4}$ & $\left(x_{1}, y_{4}\right)$ & $\left(x_{3}, y_{3}\right)$ & $D_{2}$ \\
\hline \hline$R_{3}+R_{4}$ & $\left(x_{2}, y_{1}\right)$ & $\left(x_{1}, y_{4}\right)$ & $D_{3}$ \\
\hline$R_{2}+R_{3}+R_{4}+R_{6}$ & $\left(x_{2}, y_{4}\right)$ & $\left(x_{1}, y_{2}\right)$ & $D_{4}$ \\
\hline$R_{2}+R_{3}+R_{4}+R_{5}+R_{6}$ & $\left(x_{3}, y_{3}\right)$ & $\left(x_{2}, y_{1}\right)$ & Inconsistent with idempotents. \\
\hline$R_{1}+R_{2}+R_{3}+R_{4}+R_{5}$ & $\left(x_{3}, y_{5}\right)$ & $\left(x_{2}, y_{4}\right)$ & Lost in cancelation. \\
\hline$R_{1}+R_{2}+R_{3}+R_{4}+R_{5}+R_{6}$ & $\left(x_{3}, y_{3}\right)$ & $\left(x_{2}, y_{2}\right)$ & $D_{5}$ \\
\hline \hline$R_{2}+R_{3}+2 R_{4}$ & $\left(x_{2}, y_{1}\right)$ & $\left(x_{3}, y_{3}\right)$ & Inconsistent with idempotents. \\
\hline$R_{2}+R_{3}+2 R_{4}+R_{5}$ & $\left(x_{1}, y_{2}\right)$ & $\left(x_{2}, y_{4}\right)$ & $D_{6}$ \\
\hline$R_{1}+R_{2}+R_{3}+2 R_{4}+R_{5}$ & $\left(x_{1}, y_{1}\right)$ & $\left(x_{2}, y_{4}\right)$ & Lost in cancelation. \\
\hline
\end{tabular}

TABLE 1. A summary of possible domains for the bordered diagram in Figure 1

Further, the provincial domain $R_{4}$ is a rectangle and contributes $\delta_{1}\left(x_{1}, y_{1}\right)=\left(x_{3}, y_{5}\right)$. This operation may be eliminated via the edge reduction algorithm summarized in [22, Section 2.6]. Note that this eliminates 3 more domains from consideration (as in Table 1), since the (potential) contributions to $\delta_{1}$ from each of these is lost in the cancelation.

\begin{tabular}{|l|l|l|}
\hline Domain & Contribution to $\delta_{1}$ & Sequence of Reeb chords \\
\hline \hline$D_{1}$ & $\left(x_{2}, y_{1}\right)-\rho_{1} \rightarrow\left(x_{2}, y_{2}\right)$ & $-\rho_{1}$ \\
\hline$D_{2}$ & $\left(x_{1}, y_{4}\right)-\rho_{2} \rightarrow\left(x_{3}, y_{3}\right)$ & $-\rho_{2}$ \\
\hline$D_{3}$ & $\left(x_{2}, y_{1}\right)-\rho_{3} \rightarrow\left(x_{1}, y_{4}\right)$ & $-\rho_{3}$ \\
\hline$D_{4}$ & $\left(x_{2}, y_{4}\right)-\rho_{23} \rightarrow\left(x_{1}, y_{2}\right)$ & $\left(-\rho_{2},-\rho_{3}\right)$ \\
\hline$D_{5}$ & $\left(x_{3}, y_{3}\right)-\rho_{123} \rightarrow\left(x_{2}, y_{2}\right)$ & $\left(-\rho_{1},-\rho_{2},-\rho_{3}\right)$ \\
\hline$D_{6}$ & $\left(x_{1}, y_{2}\right)-\rho_{23} \rightarrow\left(x_{2}, y_{4}\right)$ & $\left(-\rho_{2},-\rho_{3}\right)$ \\
\hline
\end{tabular}

TABLE 2. A summary of maps contributing to $\delta_{1}$ after canceling the differential corresponding to the provincial domain.

This leaves the summary of domains given in Table 2, to complete the proof we must justify the contribution from domain $D_{i}$ for $i=3,4,5,6$. From Table 2, we have

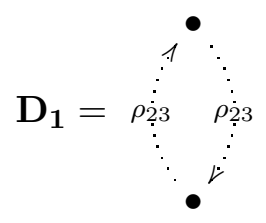


and

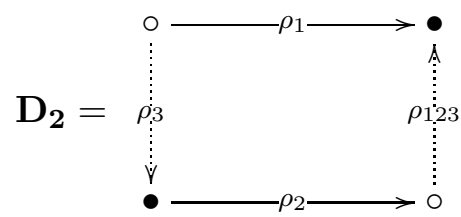

where the dotted arrows denote the 4 contributions to $\delta_{1}$ that we have yet to justify.

The domain $D_{3}$ is an annulus, containing the Reeb chord $-\rho_{3}$ in the boundary. Note that there is a single obtuse angle at $x_{1}$, and that cuts along either $\beta_{1}$ or $\alpha$ that start at $x_{1}$ decompose the annulus into a region with a single connected component (that is, such a cut meets the opposite boundary component). From this, following [22, Pages 2627] for example, we may conclude that there there are an odd number of holomorphic representatives for $D_{3}$, hence this permissible cut ensures that the domain supports a single holomorphic representative (counting modulo 2) of index 1, establishing the contribution to $\delta_{1}$.

The domain $D_{4}$ follows by similar considerations, having noted that there is a cut to the boundary starting at $y_{4}$ giving rise the the sequence $\left(-\rho_{2},-\rho_{3}\right)$ in the boundary. The obtuse angle at $x_{1}$ ensures that this annular domain supports a single holomorphic representative (counting modulo 2) of index 1 as in the previous case.

The domains $D_{5}$ and $D_{6}$ are more complicated, as each of these contains the region $R_{5}$ with multiplicity 1 . However, we may treat each of these via considerations similar to those above, having first employed the following trick.

The region $R_{5}$ may be simplified by altering the Heegaard diagram in Figure 1 by an isotopy: push the segment of $\beta_{1}$ between $x_{2}$ and $x_{3}$ to the right until a new bigon between $\alpha$ and $\beta_{1}$ is formed. Denote this new bigon by $R_{7}$ and its endpoints by $a$ and $b$ so that this new diagram produces $\delta_{1}\left(a, y_{i}\right)=\left(b, y_{i}\right)$ for $i=1,2,4$ in its corresponding type $\mathrm{D}$ structure. Note that the isotopy that removes $R_{7}$ realizes the homotopy achieved by canceling each of

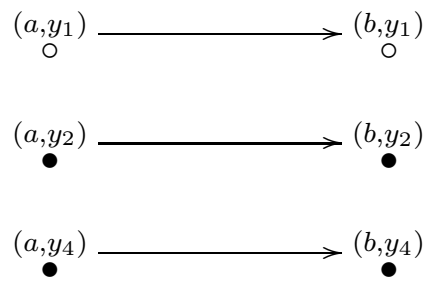

via edge reduction. Denote by $R_{5}^{+}$and $R_{5}^{-}$the two new regions formed in the top and bottom of the diagram, respectively, having performed the isotopy producing $R_{7}$. These replace the region $R_{5}$; the remaining regions are unchanged.

We could work with this enlarged diagram directly, however it will be more convenient to simply identify the decompositions of $D_{5}$ and $D_{6}$ after the isotopy. In each case, the edge in question is replaced by a unique zig-zag. 
The domain $D_{5}$ is decomposed into $D_{5}^{+}=R_{2}+R_{3}+R_{4}+R_{5}^{+}+R_{6}$ and $D_{5}^{-}=R_{1}+R_{5}^{-}$. The latter is a rectangle containing the Reeb chord $-\rho_{1}$ in the boundary, while the former is a domain with the same structure as $D_{4}$ (containing the sequence $\left(-\rho_{2},-\rho_{3}\right)$ ). This results in the zig-zag

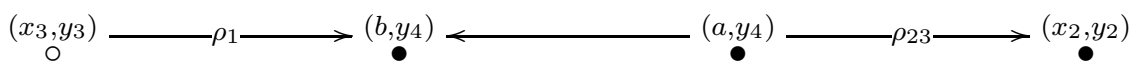

which reduces to $\stackrel{\left(x_{3}, y_{3}\right)}{\circ}-\rho_{123} \rightarrow\left(x_{2}, y_{2}\right)$ as claimed.

The domain $D_{6}$ is decomposed into $D_{6}^{+}=R_{3}+R_{4}+R_{5}^{+}$and $D_{6}^{-}=R_{2}+R_{4}+R_{5}^{-}$. Each of these is an annulus with the same structure as $D_{3}$, containing the Reeb chords $-\rho_{3}$ and $-\rho_{2}$, respectively. This results in the zig-zag

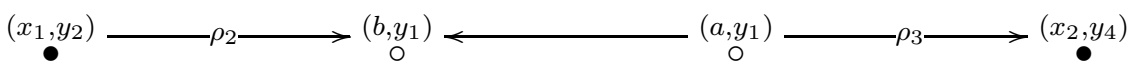

which reduces to $\left(x_{1}, y_{2}\right)-\rho_{23} \rightarrow \underset{\bullet}{\left(x_{2}, y_{4}\right)}$ as claimed.

Recall that a (right) type A structure over $\mathcal{A}$ is an $\mathbb{F}$-vector space $V$ equipped with a right action of $\mathcal{I}$ such that $V=V \iota_{0} \oplus V \iota_{1}$ and multiplication maps $m_{k+1}: V \otimes_{\mathcal{I}} \mathcal{A}^{\otimes_{\mathcal{I}} k} \rightarrow$ $V$ satisfying the $\mathcal{A}_{\infty}$ relations (see [22, Equation (2)], for example). Let $\mathbf{A}=\widehat{\mathrm{CFA}}(N)$, where $\mathbf{A}=\mathbf{A}_{\mathbf{1}} \oplus \mathbf{A}_{\mathbf{2}}$ as above. For the present purposes, it suffices to formally define $\mathbf{A}=\widehat{\mathrm{CFAA}}(\mathbb{I}, 0) \otimes \mathbf{D}$, where $\widehat{\mathrm{CFAA}}(\mathbb{I}, 0)$ is the identity type AA bimodule described in [24, Section 10.1] and summarized in [22, Section 2.4]. Considering each summand of D separately, we have $\mathbf{A}_{\mathbf{1}}=\widehat{\mathrm{CFAA}}(\mathbb{I}, 0) \otimes \mathbf{D}_{\mathbf{1}}$ and $\mathbf{A}_{\mathbf{2}}=\widehat{\mathrm{CFAA}}(\mathbb{I}, 0) \otimes \mathbf{D}_{\mathbf{2}}$. Note that this type A structure may be explicitly calculated from this information; by construction $\mathbf{A}$ is bounded.

Our conventions and those of [24, Section 10.1] ensure that

$$
\widehat{\mathrm{CF}}(W(f)) \cong\left(\mathbf{A} \otimes \mathbf{D}, \partial^{\bigotimes}\right)
$$

where $W(f)=N \cup_{f} N$ and $f$ is given by $\left(\begin{array}{ll}0 & 1 \\ 1 & 0\end{array}\right)$ (applying the main pairing result of Lipshitz, Ozsváth and Thurston [25, Theorem 1.3]). One checks, for example, that $H_{1}(W(f) ; \mathbb{Z}) \cong$ $\mathbb{Z} / 4 \oplus \mathbb{Z} / 4$ identifying the fact that $f\left(\phi_{0}\right)=\phi_{1}$ and $f\left(\phi_{1}\right)=\phi_{0}$ (the Heegaard diagram for the type A side is made explicit by appending the diagram of [24, Figure 21] to that of Figure 11). As a vector space, $\mathbf{A} \otimes \mathbf{D}$ is generated by $\mathbf{A} \otimes_{\mathcal{I}} \mathbf{D}$. Recalling that $\delta_{k}=\left(\mathrm{id}_{\mathcal{A}^{\otimes k-1}} \otimes \delta_{1}\right) \circ \delta_{k-1}$ with $\delta_{0}=\operatorname{id}_{\mathbf{D}}$, the differential is defined by

$$
\partial^{\bigotimes}(x \otimes y)=\sum_{k=0}^{\infty}\left(m_{k+1} \otimes \operatorname{id}_{\mathbf{D}}\right)\left(x \otimes \delta_{k}(y)\right)
$$

(this is well-defined since $\mathbf{A}$ is bounded). By a direct calculation one may show that $\operatorname{rk} \widehat{\mathrm{HF}}(W(f))=16$. However, as $W(f)$ is a Seifert fibred $\mathbb{Q}$-homology sphere, Theorem 4 (together with the discussion in Section 3) ensures that this manifold is an L-space and the observation about the total rank of $\widehat{\mathrm{HF}}(W(f))$ follows immediately. 
4.2. Changes of framing. Denote by $\tau_{0}$ and $\tau_{1}$ the Dehn twists along $\phi_{0}$ and $\phi_{1}$, respectively. In [24, Section 10.2], the type DA bimodules corresponding to these mapping classes are described, and these may be composed via the box tensor product to change the framing on D. We note that our conventions are such that the Dehn twists $\tau_{0}$ and $\tau_{1}$ correspond to the Dehn twists $\tau_{m}$ and $\tau_{l}$, respectively, in [24, Section 10.2]. This can be seen, for example, by considering the effect on the peripheral elements in the new bordered Heegaard diagram obtained by adjoining each of the diagrams of [24, Figure 25] to the boundary of the diagram in Figure 1 (realizing the change of framing).

Let $\mathbf{T}_{\mathbf{0}}=\widehat{\mathrm{CFDA}}\left(\tau_{0}, 0\right)$. As has been noted above, our conventions ensure that

$$
\widehat{\mathrm{CF}}(W(f)) \cong \mathbf{A} \otimes \mathbf{D}
$$

where $f$ is given by $\left(\begin{array}{ll}0 & 1 \\ 1 & 0\end{array}\right)$. As a result, $\mathbf{A} \otimes \mathbf{T}_{\mathbf{0}}^{\bigotimes n} \otimes \mathbf{D}$ gives a complex $\widehat{\mathrm{CF}}(W(f))$ for the manifold $W(f)=N \cup_{f} N$ where the homeomorphism $f$ is specified by $\left(\begin{array}{ll}n & 1 \\ 1 & 0\end{array}\right)=\left(\begin{array}{ll}1 & n \\ 0 & 1\end{array}\right)\left(\begin{array}{ll}0 & 1 \\ 1 & 0\end{array}\right)$ for any integer $n$. We interpret negative values for $n$ via $\mathbf{T}_{\mathbf{0}}^{\bigotimes(-1)}=\widehat{\operatorname{CFDA}}\left(\tau_{0}^{-1}, 0\right)$, so that for any integer $n$ we get a type $\mathbf{D}$ structure $\mathbf{T}_{\mathbf{0}}^{\bigotimes n} \otimes \mathbf{D}$.

Proposition 21. For all $n \in \mathbb{Z}$ we have

$$
\mathbf{T}_{\mathbf{0}}^{\otimes n} \otimes \mathbf{D} \cong \mathbf{D}
$$

where $\cong$ denotes chain homotopy equivalence of type D structures.

Proof. A description of $\mathbf{T}_{\mathbf{0}}$ is given in [24, Figure 27] via a directed graph; we will record only the subgraph relevant to the present calculations.

First consider the summand $\mathbf{D}_{\mathbf{1}}$. Since $\iota_{0} \mathbf{D}_{\mathbf{1}}=0$, we need only consider generators of $\mathbf{T}_{\mathbf{0}} \iota_{1}$ (and relevant operations relating them). In particular, we have that $\mathbf{T}_{\mathbf{0}} \iota_{1}$ is described by the directed graph

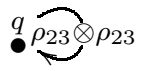

where the label $\rho_{23} \otimes \rho_{23}$ indicates that $m\left(q, \rho_{23}\right)=\rho_{23} \otimes q(q$ is the single generator for which $\left.\iota_{1} q \iota_{1}=q\right)$. From this it is immediate that $\mathbf{T}_{\mathbf{0}} \otimes \mathbf{D}_{\mathbf{1}}=\mathbf{D}_{\mathbf{1}}$ hence $\mathbf{T}_{\mathbf{0}}^{\otimes n} \otimes \mathbf{D}_{\mathbf{1}}=\mathbf{D}_{\mathbf{1}}$.

Next consider the summand $\mathbf{D}_{\mathbf{2}}$. Since neither elements $\rho_{12}$ nor $\rho_{23}$ appear in $\mathbf{D}_{\mathbf{2}}$, operations involving $\rho_{12}$ or $\rho_{23}$ on the right will not be used in the box tensor product when calculating $\delta_{1}$ for the type D structure $\mathbf{T}_{\mathbf{0}} \otimes \mathbf{D}_{\mathbf{2}}$. As a result, the relevant operations of $\mathbf{T}_{\mathbf{0}}$ are described by the directed graph

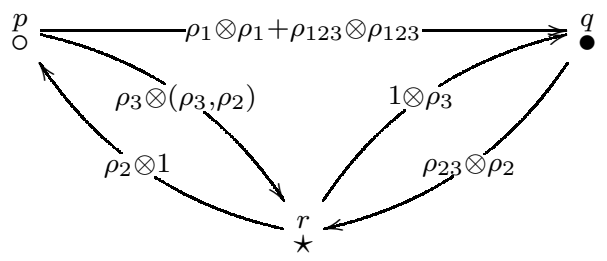

where, for example, the edge labelled $\rho_{3} \otimes\left(\rho_{3}, \rho_{2}\right)$ indicates that $m\left(p, \rho_{3}, \rho_{2}\right)$ contains a summand $\rho_{3} \otimes r$. The action of $\mathcal{I}$ on the generators is given by $\iota_{0} p \iota_{0}=p$ (denoted by $\circ$ ), $\iota_{1} q \iota_{1}=q$ (denoted by $\left.\bullet\right)$, and $\iota_{1} r \iota_{0}=r$ (denoted by $\left.\star\right)$. The notation $\star$ is intended to 
indicate the change from $\circ$ to $\bullet$ when calculating the box tensor product with a type D structure. Note that, for any element marked $\circ$ (an element in the $\iota_{0}$-summand) in a type $\mathrm{D}$ structure we have that $\mathbf{T}_{\mathbf{0}} \boldsymbol{\nabla}$ - produces an edge $\bullet-\rho_{2} \rightarrow 0$ in the new type D structure. Now the box tensor product
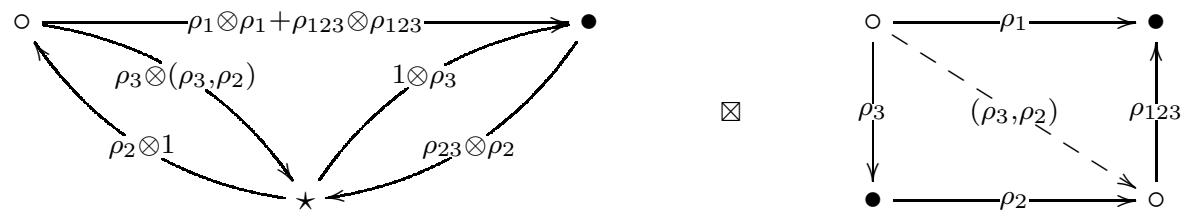

gives the type $\mathrm{D}$ structure of interest; the dashed arrow labelled $\left(\rho_{3}, \rho_{2}\right)$ indicates the operation $\delta_{2}(x)=\rho_{3} \otimes \rho_{2} \otimes y$ for generators $x, y \in \iota_{0} \mathbf{D}_{\mathbf{2}}$. As a result $\mathbf{T}_{\mathbf{0}} \otimes \mathbf{D}_{\mathbf{2}}$ is described by the directed graph

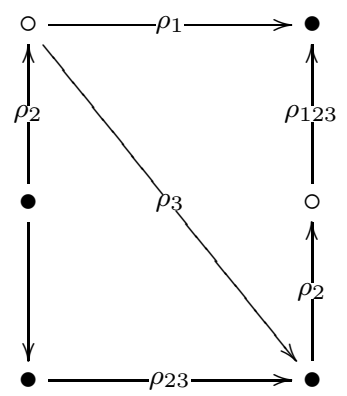

By edge reduction, this is chain homotopy equivalent to $\mathbf{D}_{\mathbf{2}}$ as claimed.

Combining these two calculations gives $\mathbf{T}_{\mathbf{0}} \otimes \mathbf{D} \cong \mathbf{D}$, and this may be iterated to obtain $\mathbf{T}_{\mathbf{0}}^{\bigotimes n} \otimes \mathbf{D} \cong \mathbf{D}$ for $n>0$. A similar calculation yields the case $n<0$.

By contrast, setting $\mathbf{T}_{\mathbf{1}}=\widehat{\mathrm{CFDA}}\left(\tau_{1}, 0\right)$ we have that $\widehat{\mathrm{CF}}(W(f)) \cong \mathbf{A} \otimes \mathbf{T}_{\mathbf{1}}^{\otimes n} \otimes \mathbf{D}$ for the torus semi-bundle $W(f)$ arising from identification via any homeomorphism $f$ of the form $\left(\begin{array}{ll}0 & 1 \\ 1 & n\end{array}\right)=\left(\begin{array}{ll}1 & 0 \\ n & 1\end{array}\right)\left(\begin{array}{ll}0 & 1 \\ 1 & 0\end{array}\right)$. Again, we denote $\mathbf{T}_{\mathbf{1}}^{\bigotimes(-1)}=\widehat{\mathrm{CFDA}}\left(\tau_{1}^{-1}, 0\right)$. In this setting $\mathbf{T}_{1}^{\bigotimes n} \otimes-$ is non-trivial (in the sense of Proposition 21) when applied to $\mathbf{D}$. The structure of $\mathbf{T}_{1}^{\bigotimes n} \otimes \mathbf{D}$ is well-behaved, and may be easily computed (proceeding as in the argument above) for any $n \in \mathbb{Z}$. However, this will not be needed (explicitly) in the present setting, so we leave it as an exercise for the interested reader.

4.3. An infinite family of L-spaces of rank 16: the base case. For the homeomorphism $g$ described by $\left(\begin{array}{ll}1 & a \\ 0 & 1\end{array}\right)\left(\begin{array}{ll}1 & 0 \\ b & 1\end{array}\right)\left(\begin{array}{ll}0 & 1 \\ 1 & 0\end{array}\right)=\left(\begin{array}{cc}a & a b+1 \\ 1 & b\end{array}\right)$ we have

$$
\widehat{\mathrm{CF}}(W(g)) \cong \mathbf{A} \otimes \mathbf{T}_{\mathbf{1}}^{\otimes b} \otimes \mathbf{T}_{\mathbf{0}}^{\bigotimes a} \otimes \mathbf{D}
$$

with $W(g)=N \cup_{g} N$, for all $a, b \in \mathbb{Z}$. This family of torus semi-bundles is of immediate interest.

Theorem 22. Let $N$ be the twisted I-bundle over the Klein bottle. Let $g$ be the homeomorphism $\partial N \rightarrow \partial N$ defined by the matrix $\left(\begin{array}{c}a \\ 1 \\ a b+1 \\ b\end{array}\right)$ for any $a, b \in \mathbb{Z}$. Then the torus semi-bundle $W(g)=N \cup_{g} N$ is an L-space, with $\operatorname{rk} \widehat{\mathrm{HF}}(W(g))=16$. 
Note that when either $a=0$ or $b=0$, the resulting manifold is a Seifert fibered space. In this special case Theorem 22 follows from work of Boyer, Rolfsen and Wiest [3] (see the discussion in Section 3) and Theorem 4. Generically however, $W(g)=N \cup_{g} N$ is a Sol manifold, and it is this case that is of present interest.

Proof of Theorem 22. It suffices to prove that $\operatorname{rk} H_{*}\left(\mathbf{A} \otimes \mathbf{T}_{\mathbf{1}}^{\otimes b} \otimes \mathbf{T}_{\mathbf{0}}^{\bigotimes a} \otimes \mathbf{D}\right)=16$. By Proposition 21,

$$
\mathbf{T}_{\mathbf{1}}^{\bigotimes b} \otimes \mathbf{T}_{\mathbf{0}}^{\bigotimes a} \otimes \mathbf{D} \cong \mathbf{T}_{1}^{\otimes b} \otimes \mathbf{D}
$$

as type $\mathrm{D}$ structures, so that

$$
H_{*}\left(\mathbf{A} \otimes \mathbf{T}_{\mathbf{1}}^{\bigotimes b} \otimes \mathbf{T}_{\mathbf{0}}^{\bigotimes a} \otimes \mathbf{D}\right) \cong H_{*}\left(\mathbf{A} \otimes \mathbf{T}_{\mathbf{1}}^{\otimes b} \otimes \mathbf{D}\right)
$$

Recall that $H_{*}\left(\mathbf{A} \otimes \mathbf{T}_{1}^{\otimes b} \otimes \mathbf{D}\right) \cong \widehat{\mathrm{HF}}(W(g))$ where $g$ is the homeomorphism defined by $\left(\begin{array}{ll}0 & 1 \\ 1 & b\end{array}\right)$. As previously observed, this is a Seifert fibred L-space with $\left|H_{1}(W(g) ; \mathbb{Z})\right|=16$, hence $\mathrm{rk} \widehat{\mathrm{HF}}(W(g))=16$ as claimed.

The key feature of this argument is the (more general) observation that post-composing any homeomorphim by $\tau_{0}^{n}$ gives $\left(\begin{array}{ll}1 & n \\ 0 & 1\end{array}\right)\left(\begin{array}{ll}a & b \\ c & d\end{array}\right)=\left(\begin{array}{cc}a+n c & b+n d \\ c & d\end{array}\right)$; Proposition 21 implies that the (ungraded) Heegaard Floer homology is identical for the family of torus semi-bundles obtained via these homeomorphisms. The proof of Theorem 22 makes use of the fact that for $c=1$, choosing $n=-a$ yields a Seifert fibred torus semi-bundle.

\section{The inductive Step in the Proof of Theorem 5}

In this section we complete the proof of Theorem [5 with the following proposition (cf. Theorem 221).

Proposition 23. Suppose that $W(f)$ is an L-space whenever $f_{*}=\left(\begin{array}{ll}a & b \\ c & d\end{array}\right)$ where $c=1$ and $\operatorname{det}\left(f_{*}\right)=-1$. Then $W(f)$ is an L-space whenever $c \neq 0$.

The proof is at the end of this section. To set it up, let $f$ be a homeomorphism of $T$ with matrix $f_{*}=\left(\begin{array}{ll}a & b \\ c & d\end{array}\right)$ with respect to $\phi_{0}, \phi_{1}$. Our first goal is to understand conditions under which Dehn surgery on $W(f)$ along a knot contained in $T=\partial N$ yields an L-space.

Let $\gamma$ be a slope on $T$ represented by a simple closed curve $K_{\gamma} \subset T \subset W(f)$. As $T$ is oriented, we have a homeomorphism $D_{K_{\gamma}}: T \rightarrow T$, well-defined up to isotopy, given by a Dehn twist along $K_{\gamma}$. On the level of homology

$$
\left(D_{K_{\gamma}}\right)_{*}(\beta)=\beta+(\beta \cdot \gamma) \gamma \text { for all } \beta \in H_{1}(T)
$$

Denote the exterior of $K_{\gamma}$ in $W(f)$ by $M_{f, \gamma}$ and set $T_{\gamma}=\partial M_{f, \gamma}$. There is a basis $\left\{\mu_{\gamma}, \lambda_{T}\right\}$ of $H_{1}\left(T_{\gamma}\right)$ where $\mu_{\gamma}$ is a meridian of $K_{\gamma}$ and $\lambda_{T}$ is represented by a parallel of $K_{\gamma}$ lying on $T$. Orient $\lambda_{T}$ and $\mu_{\gamma}$ so that $\mu_{\gamma} \cdot \lambda_{T}=1$ with respect to the induced orientation on $T_{\gamma}$. Our first goal is to determine the constant $\epsilon(f, \gamma)$ such that $\left(\mu_{\gamma}, \epsilon(f, \gamma) \lambda_{T}, \mu_{\gamma}+\epsilon(f, \gamma) \lambda_{T}\right)$ is a triad (cf. Definition 15). 
Note that

$$
M_{f, \gamma}\left(\mu_{\gamma}\right)=W(f)
$$

while

$$
M_{f, \gamma}\left(\lambda_{T}\right)=N(\gamma) \# N\left(f_{*}(\gamma)\right)
$$

The latter is an $L$-space as long as neither $\gamma$ nor $f_{*}(\gamma)$ is $\pm \phi_{0}$. More precisely, let

$$
\gamma=r \phi_{0}+s \phi_{1} \quad \text { and } \quad f_{*}(\gamma)=u \phi_{0}+v \phi_{1}
$$

Then

$$
u=a r+b s \quad \text { and } \quad v=c r+d s
$$

The only filling of $N$ which is not an $L$-space is the $\phi_{0}$-filling. Further $\left|H_{1}(N(\gamma))\right|=$ $4 \Delta\left(\gamma, \phi_{0}\right)=4|s|$ and $\left|H_{1}\left(N\left(f_{*}(\gamma)\right)\right)\right|=4|v|$. Thus $M_{f, \gamma}\left(\lambda_{T}\right)$ is an $L$-space if and only if $s, v \neq 0$. In this case,

$$
\left|H_{1}\left(M_{f, \gamma}\left(\lambda_{T}\right)\right)\right|=16|s v|
$$

For $c, s, v \neq 0$ set

$$
\epsilon(f, \gamma)=-\operatorname{sign}(c s v)
$$

and note that $\left|H_{1}\left(M_{f, \gamma}\left(\mu_{\gamma}+\epsilon(f, \gamma) \lambda_{T}\right)\right)\right|=\left|H_{1}\left(M_{f, \gamma}\left(\mu_{\gamma}\right)\right)\right|+\left|H_{1}\left(M_{f, \gamma}\left(\lambda_{T}\right)\right)\right|$. Consequently $\left(\mu_{\gamma}, \epsilon(f, \gamma) \lambda_{T}, \mu_{\gamma}+\epsilon(f, \gamma) \lambda_{T}\right)$ is a triad.

It is well-known that $M_{f, \gamma}\left(\mu_{\gamma}+n \lambda_{T}\right)=W\left(g_{n}\right)$ where $g_{n}=f \circ D_{K_{\gamma}}^{-n}$. In particular, $\left(g_{n}\right)_{*}(\beta)=$ $f_{*}(\beta)-n(\beta \cdot \gamma) f_{*}(\gamma)$. Hence $\left(g_{n}\right)_{*}=\left(\begin{array}{ll}a-n s u & b+n r u \\ c-n s v & d+n r v\end{array}\right)$ and therefore

Lemma 24. Suppose that $W(f)$ is an L-space and $\gamma, f_{*}(\gamma) \neq \pm \phi_{0}$. Then $W\left(g_{n}\right)$ is an $L$-space for all $n \geq 0$ where $g_{n}=f \circ D_{K_{\gamma}}^{-\epsilon(f, \gamma) n}$. Further, if $f_{*}=\left(\begin{array}{ll}a & b \\ c & d\end{array}\right)$ then

$$
\left(g_{n}\right)_{*}=\left(\begin{array}{ll}
a-n \epsilon(f, \gamma) s u & b+n \epsilon(f, \gamma) r u \\
c-n \epsilon(f, \gamma) s v & d+n \epsilon(f, \gamma) r v
\end{array}\right)
$$

Lemma 25. Let $f$ and $\gamma$ be as above and let $n \geq 0$. Suppose that $W(g)$ is an L-space whenever $g_{*}$ has first column $\left(\begin{array}{c}a \\ c\end{array}\right)$. Then $W(h)$ is an L-space whenever $h_{*}$ has first column $\left(\begin{array}{c}a-n \epsilon(f, \gamma) s u \\ c-n \epsilon(f, \gamma) s v\end{array}\right)$.

Proof. Consider a homeomorphism $h$ of $T$ such that $h_{*}$ has first column $\left(\begin{array}{l}a-n \epsilon(f, \gamma) s u \\ c-n \epsilon(f, \gamma) s v\end{array}\right)$. To see that $W(h)$ is an L-space we can suppose that $\operatorname{det}\left(h_{*}\right)=\operatorname{det}\left(f_{*}\right)$ by Remark 19. Then Lemma 24 implies that the second column of $h_{*}$ can then be written

$$
\left(\begin{array}{l}
b^{\prime} \\
d^{\prime}
\end{array}\right)=\left(\begin{array}{l}
b+n \epsilon(f, \gamma) r u \\
d+n \epsilon(f, \gamma) r v
\end{array}\right)+m\left(\begin{array}{l}
a-n \epsilon(f, \gamma) s u \\
c-n \epsilon(f, \gamma) s v
\end{array}\right)=\left(\begin{array}{c}
b+m a \\
d+m c
\end{array}\right)+n \epsilon(f, \gamma)(r-m s)\left(\begin{array}{l}
u \\
v
\end{array}\right)
$$

for some $m \in \mathbb{Z}$.

By hypothesis, $W(k)$ is an L-space where $k$ is the homeomorphism of $T$ with matrix $k_{*}=$ $\left(\begin{array}{ll}a & b+m a \\ c & d+m c\end{array}\right)$. Let $\gamma^{\prime}=r^{\prime} \phi_{0}+s \phi_{1}$ where $r^{\prime}=r-m s$. Then $k_{*}\left(\gamma^{\prime}\right)=(a(r-m s)+(b+$ $m a) s) \phi_{0}+(c(r-m s)+(d+m c) s) \phi_{1}=(a r+b s) \phi_{0}+(c r+d s) \phi_{1}=u \phi_{0}+v \phi_{1}=f_{*}(\gamma)$. 
Hence $\gamma^{\prime}, k_{*}\left(\gamma^{\prime}\right) \neq \pm \phi_{0}$. Further note that $\epsilon\left(k, \gamma^{\prime}\right)=-\operatorname{sign}(c s v)=\epsilon(f, \gamma)$. Lemma 24 then shows that $W\left(k_{n}\right)$ is an L-space where

$$
\left(k_{n}\right)_{*}=\left(\begin{array}{ll}
a-n \epsilon(f, \gamma) s u & b+m a+n \epsilon(f, \gamma) r^{\prime} u \\
c-n \epsilon(f, \gamma) s v & d+m c+n \epsilon(f, \gamma) r^{\prime} v
\end{array}\right)
$$

The second column of $\left(k_{n}\right)_{*}$ is

$$
\left(\begin{array}{l}
b+m a+n \epsilon(f, \gamma) r^{\prime} u \\
d+m c+n \epsilon(f, \gamma) r^{\prime} v
\end{array}\right)=\left(\begin{array}{l}
b+m a \\
d+m c
\end{array}\right)+n \epsilon(f, \gamma)(r-m s)\left(\begin{array}{l}
u \\
v
\end{array}\right)=\left(\begin{array}{l}
b^{\prime} \\
d^{\prime}
\end{array}\right)
$$

Hence $\left(k_{n}\right)_{*}=h_{*}$, which completes the proof.

Proof of Proposition 23. Consider a torus semi-bundle $W(f)$ where $f_{*}=\left(\begin{array}{ll}a & b \\ c & d\end{array}\right)$ and $c \neq 0$. We will show that $W(f)$ is an L-space assuming that this is the case when $c=1$ and $\operatorname{det}\left(f_{*}\right)=-1$. By Remark 19, this assumption implies that $W(f)$ is an L-space whenever $|c|=1$. We proceed by induction on $|c|$.

Let $C$ be an integer of absolute value 2 or larger and suppose that $W(f)$ is an L-space whenever $f_{*}=\left(\begin{array}{ll}a & b \\ c & d\end{array}\right)$ where $1 \leq|c|<|C|$. Consider a torus semi-bundle $W(F)$ where $F_{*}=\left(\begin{array}{ll}A & B \\ C & D\end{array}\right)$. By Remark 19 we can suppose that $C>1$. Choose integers $a, c$ such that $a C-c A=1$ and $0<c<C$. Set $b=A-2 a, d=C-2 c$. Then $a d-b c=a C-c A=1$ so $f_{*}=\left(\begin{array}{ll}a & b \\ c & d\end{array}\right) \in S L_{2}(\mathbb{Z})$. By induction, $W(g)$ is an L-space for all $g$ such that $g_{*}$ has first column $\left(\begin{array}{l}a \\ c\end{array}\right)$.

Take $\gamma=\phi_{0}+\phi_{1}$ so that $f_{*}(\gamma)=(a+b) \phi_{0}+(c+d) \phi_{1}$. Then in the notation established earlier in this section, $u=a+b$ and $v=c+d=C-c>0$. Hence $\epsilon(f, \gamma)=-\operatorname{sign}(\operatorname{cs} v)=-1$ so that Lemma 25 implies that $W(G)$ is an L-space for all $G$ such that $G_{*}$ has first column $\left(\begin{array}{c}a-\epsilon(f, \gamma) s u \\ c-\epsilon(f, \gamma) s v\end{array}\right)=\left(\begin{array}{c}a+(a+b) \\ c+(c+d)\end{array}\right)=\left(\begin{array}{c}A \\ C\end{array}\right)$. In particular $W(F)$ is an L-space. This completes the induction.

\section{2-FOLD BRANCHED COVERS OF ALTERNATING LINKS}

In this section we prove Theorem 8 .

6.1. Wada's group. Let $L$ be a link in $S^{3}$ and $D$ a diagram for $L$. Label the arcs of the diagram 1 through $n$ as in Figure 2. Define a group $\pi(D)$ as follows: $\pi(D)$ has generators $a_{1}, a_{2}, \ldots, a_{n}$ in one-one correspondence with the $\operatorname{arcs}$ of $D$, and relations of the form

$$
a_{k} a_{j}^{-1} a_{i} a_{j}^{-1} \text {. }
$$

in one-one correspondence with the crossings of $D$. Note that this relation is well-defined, as it is invariant under interchanging the indices $i$ and $k$.

This presentation was considered by Wada [46], who proved the following theorem. (See also [41.) We include a proof for completeness.

Theorem 26. $\pi(D) \cong \pi_{1}(\Sigma(L)) * \mathbb{Z}$ where $\Sigma(L)$ is the 2-fold branched cover of $L$. 

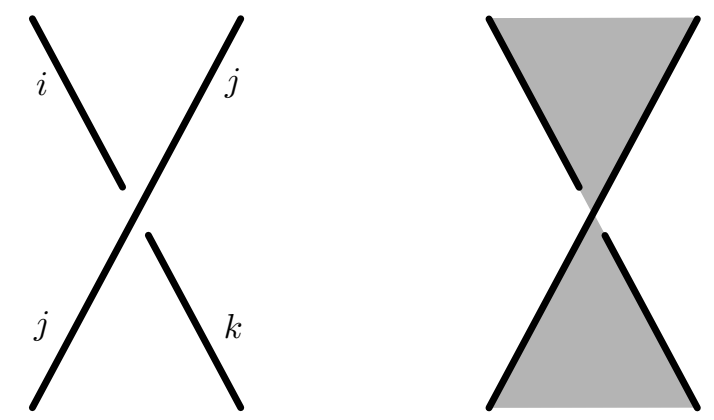

FiguRE 2. Labeling the arcs at a crossing (left), and the checkerboard convention for the black graph (right).

Proof. Let $M_{L}$ be the complement of $L$. The Wirtinger presentation of $\pi_{1}\left(M_{L}\right)$ corresponding to the diagram $D$ has generators $a_{1}, a_{2}, \ldots, a_{n}$ as above and a relation at each crossing, of the form

$$
a_{k}^{-1} a_{j} a_{i} a_{j}^{-1}
$$

for suitable choice of labels $i$ and $k$. Let $X$ be the 2-complex of this presentation, with one 0 -cell $x, n$ oriented 1-cells, and $n$ 2-cells. Thus $\pi_{1}(X) \cong \pi_{1}\left(M_{L}\right)$. Let $\widetilde{X}$ be the (connected) double cover of $X$ determined by the homomorphism $\pi_{1}(X) \rightarrow \mathbb{Z} / 2, a_{i} \mapsto 1$ for all $i$. In $\widetilde{X}$ the 0 -cell $x$ of $X$ lifts to two 0 -cells $x_{1}$ and $x_{2}$, and each 1 -cell $a_{i}$, say, of $X$ lifts to two 1-cells that we will denote by $\alpha_{i}$ and $\beta_{i}$, where $\alpha_{i}$ is oriented from $x_{1}$ to $x_{2}$ and $\beta_{i}$ from $x_{2}$ to $x_{1}$. Let $\widetilde{X}_{+}$be obtained from $\widetilde{X}$ by adjoining an arc $e$, identifying $\partial e$ with $\left\{x_{1}, x_{2}\right\}$. Then $\pi_{1}\left(\widetilde{X}_{+}\right) \cong \pi_{1}(\widetilde{X}) * \mathbb{Z}$. Taking as "base-point" for $\widetilde{X}_{+}$the maximal tree $e$ we obtain the following presentation for $\pi_{1}\left(\widetilde{X}_{+}\right)$: generators $\alpha_{1}, \beta_{1}, \alpha_{2}, \beta_{2}, \ldots, \alpha_{n}, \beta_{n}$ and pairs of relations, corresponding to the two lifts of each 2-cell in $X$,

$$
\begin{aligned}
& \beta_{k}^{-1} \beta_{j} \alpha_{i} \alpha_{j}^{-1} \\
& \alpha_{k}^{-1} \alpha_{j} \beta_{i} \beta_{j}^{-1}
\end{aligned}
$$

Since $\pi_{1}(\widetilde{X}) \cong \pi_{1}\left(\widetilde{M}_{L}\right)$, where $\widetilde{M}_{L}$ is the double cover of $M_{L}$, and since $a_{1}, a_{2}, \ldots, a_{n}$ are meridians of $L$, we obtain a presentation for $\pi_{1}(\Sigma(L)) * \mathbb{Z}$ by adding the branching relations

$$
\alpha_{1} \beta_{1}=\alpha_{2} \beta_{2}=\ldots=\alpha_{n} \beta_{n}=1
$$

Thus eliminating $\beta_{1}=\alpha_{1}^{-1}, \beta_{2}=\alpha_{2}^{-1}, \ldots, \beta_{n}=\alpha_{n}^{-1}$, equations (3) and (4) become

$$
\begin{aligned}
& \alpha_{k} \alpha_{j}^{-1} \alpha_{i} \alpha_{j}^{-1} \\
& \alpha_{k}^{-1} \alpha_{j} \alpha_{i}^{-1} \alpha_{j}
\end{aligned}
$$

Since the second relation is a consequence of the first, those relations may be eliminated. This gives the presentation of $\pi(D)$ defined above. 
6.2. The proof of Theorem $\mathbf{8}$, Let $L$ be an alternating link. We begin by reducing the proof of the theorem to the case where $L$ is non-split.

Suppose that $L$ is split and Theorem 8 holds for non-split alternating links. The fundamental group of the 2-fold cover of $S^{3}$ branched over $L$ is of the form

$$
\pi_{1}(\Sigma(L)) \cong F_{n-1} * \pi_{1}\left(\Sigma\left(L_{1}\right)\right) * \pi_{1}\left(\Sigma\left(L_{2}\right)\right) * \ldots * \pi_{1}\left(\Sigma\left(L_{n}\right)\right)
$$

where $n \geq 2, F_{n-1}$ is free of rank $n-1$, and $L_{1}, L_{2}, \ldots, L_{n}$ are non-split alternating links. By assumption, $\pi_{1}\left(\Sigma\left(L_{j}\right)\right)$ is not left-orderable for each $j$. Hence if $\pi_{1}(\Sigma(L))$ is left-orderable, each $\pi_{1}\left(\Sigma\left(L_{j}\right)\right)$ is the trivial group. It follows that each $L_{j}$ is a trivial knot and therefore $L$ is a trivial link of two or more components, so Theorem 8 holds.

Assume next that $L$ is non-split and let $D$ be an alternating diagram for $L$. Label its arcs 1 through $n$ and note that the crossings of $D$ correspond somewhat ambiguously to ordered label triples $(i, j, k)$ where $j$ is the label of the overcrossing arc. (Thus $(i, j, k)$ and $(k, j, i)$ represent the same crossing.)

Theorem 8 clearly holds when $L$ is the trivial knot so we suppose below that it isn't. Then $\pi_{1}(\Sigma(L))$ is non-trivial so that $\pi(D) \cong \pi_{1}(\Sigma(L)) * \mathbb{Z}$ is not abelian. Vinogradov proved that the free product of two non-trivial groups is left-orderable if and only if the two factors are left-orderable [45]. Thus $\pi_{1}(\Sigma(L))$ is left-orderable if and only if $\pi(D) \cong \pi_{1}(\Sigma(L)) * \mathbb{Z}$ is left-orderable, so the theorem will follow if we show that the hypothesis that $\pi(D)$ is left-orderable implies that $\pi(D)$ is abelian. Suppose then that " $<$ " is a left-ordering on $\pi(D)$.

Consider the black-white checkerboard pattern on $S^{2}$ determined by $D$ where we assume that the black regions lie to the left as we pass over a crossing. (This convention is illustrated in Figure 2, )

Fix a crossing $(i, j, k)$. Relation (1) shows that $a_{j}^{-1} a_{i}=\left(a_{j}^{-1} a_{k}\right)^{-1}$. It follows that exactly one of the following three possibilities occurs:

$$
\begin{aligned}
& a_{i}<a_{j}<a_{k} \\
& a_{k}<a_{j}<a_{i} \\
& a_{i}=a_{j}=a_{k}
\end{aligned}
$$

We use these options to define a semi-oriented graph $\Gamma(D)$ in $S^{2}$ as follows: the vertices of $\Gamma(D)$ correspond to the black regions of $D$, the edges $e$ correspond to the crossings of $D$, and the embedding in $S^{2}$ is that determined by $D$. Note that $\Gamma(D)$ is connected as $L$ is non-split.

Fix an edge $e=e(i, j, k)$ and let $R_{i}, R_{k}$ be the black regions containing the arcs labelled $i, k$ respectively. Orient $e$ from $R_{i}$ to $R_{k}$ if possibility (5) occurs, from $R_{k}$ to $R_{i}$ if (6) occurs, and do not orient it if (7) occurs (see Figure 3). 

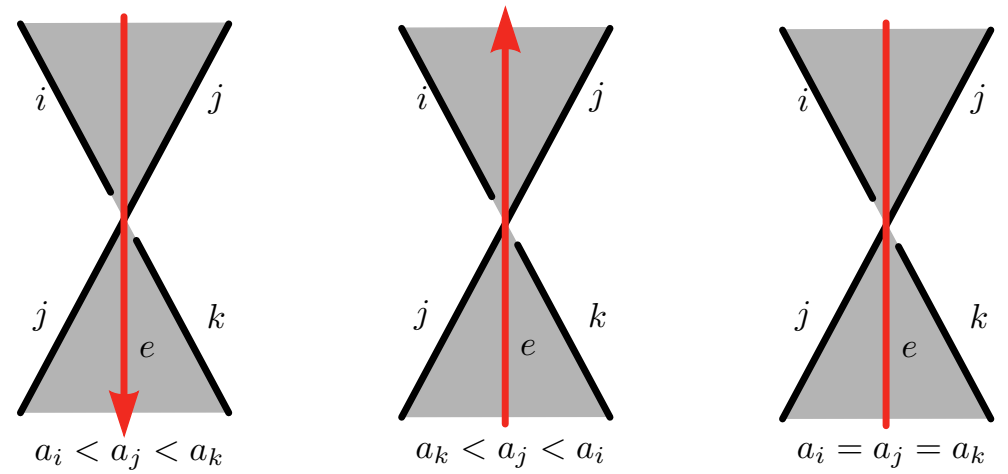

Figure 3. Orientation conventions for the edges of the semi-oriented graph $\Gamma(D)$.

A circuit in $\Gamma(D)$ is a simple closed curve in $S^{2}$ determined by a sequence of edges $C$ : $e_{1}, e_{2}, \ldots, e_{m}$ of $\Gamma(D)$ indexed so that successive edges are incident, and those edges of $C$ which are oriented, are oriented coherently.

A cycle in $\Gamma(D)$ is an innermost circuit in $\Gamma(D)$. Equivalently it is a circuit which bounds a white region of $D$.

Lemma 27. Each edge contained in a cycle of $\Gamma(D)$ is unoriented.

Proof. Suppose that $\Gamma(D)$ contains a cycle and let $R$ be the white region of $D$ it determines. We can label its boundary edges $i_{1}, i_{2}, \ldots, i_{r}$ so that the cycle is given, up to reversing its order, by the sequence of crossings $\left(i_{1}, i_{2}, k_{1}\right),\left(i_{2}, i_{3}, k_{2}\right), \ldots,\left(i_{r}, i_{1}, k_{r}\right)$ (see Figure 4). The cycle condition implies that either

$$
a_{i_{1}} \leq a_{i_{2}} \leq \ldots \leq a_{i_{r}} \leq a_{i_{1}}
$$

or

$$
a_{i_{1}} \geq a_{i_{2}} \geq \ldots \geq a_{i_{r}} \geq a_{i_{1}}
$$

Thus all inequalities are equalities, so none of the edges of the cycle are oriented.

A sink, respectively source, of $\Gamma(D)$ is a vertex $v$ of $\Gamma(D)$ such that each oriented edge of $\Gamma(D)$ incident to $v$ points into, respectively away from, $R$.

Lemma 28. Each edge incident to a source or sink in $\Gamma(D)$ is unoriented.

Proof. Let $R$ be a black region determined by $D$ and let $i_{1}, i_{2}, \ldots, i_{r}$ be the labels of its boundary arcs indexed $(\bmod r)$ so that the crossings of $D$ incident to $R$ are determined by the black corners $\left(i_{1}, i_{2}\right),\left(i_{2}, i_{3}\right), \ldots,\left(i_{r}, i_{1}\right)$ (see Figure 4).

Then $a_{i_{1}} \leq a_{i_{2}} \leq \ldots \leq a_{i_{r}} \leq a_{i_{1}}$ if the vertex $v$ is a sink, while $a_{i_{1}} \geq a_{i_{2}} \geq \ldots \geq a_{i_{r}} \geq a_{i_{1}}$ if it is a source. In either case, $a_{i_{1}}=a_{i_{2}}=\ldots=a_{i_{r}}$, so no edge incident to $v$ is oriented.

On the other hand, we have the following result. 

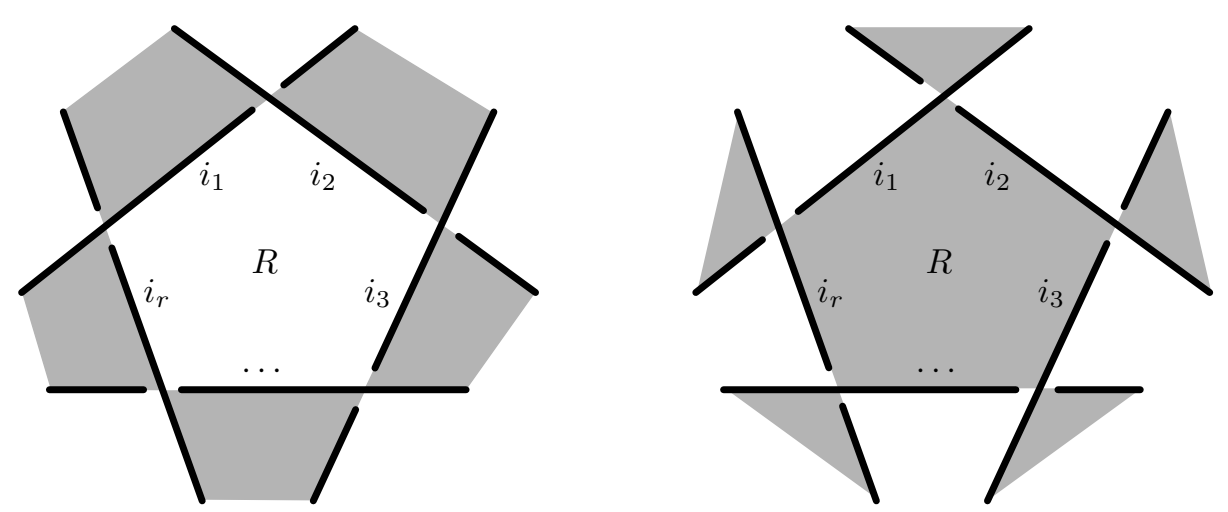

FiguRE 4. White and black regions of the checkerboard colouring considered in the proofs of Lemma 27 and Lemma 28, respecively.

Lemma 29. Let $\Gamma$ be a connected semi-oriented graph in $S^{2}$ without sinks or sources containing oriented edges. If some edge e of $\Gamma$ is oriented, then there is a cycle of $\Gamma$ containing an oriented edge.

Proof. Since $\Gamma$ has no sinks, the vertex at the head of an oriented edge is incident to the tail of another oriented edge of $\Gamma$. Starting from some oriented edge, we obtain a first return circuit, all of whose edges are oriented.

Choose a circuit $C$ in $\Gamma$ which is innermost among the family of circuits all of whose edges are oriented. Then $C$ bounds a disk $E$ such that each circuit $C^{\prime} \neq C$ contained in $\Gamma \cap E$ has an unoriented edge. Suppose some edge $e$ of $(\Gamma \cap E) \backslash C$ is oriented. Arguing as in the first paragraph of this proof, and using our innermost assumption on $C$, we obtain an oriented path of edges starting with $e$ and ending at some vertex in $C$. Similarly, since $\Gamma$ has no sources, we can carry out the same construction backwards, starting from the tail of $e$. This produces a (non-empty) oriented path of edges in $(\Gamma \cap E) \backslash C$ starting and ending on $C$, which in turn gives a circuit of oriented edges that contradicts the innermost property of $C$. Thus each edge of $\Gamma \backslash C$ contained in $E$ is unoriented. It follows that the boundary of any region of $D$ contained in $E$ whose boundary contains an edge of $C$ determines a cycle containing an oriented edge.

The last three lemmas combine to show that each edge of $\Gamma(D)$ is unoriented. It follows that for each crossing $(i, j, k)$,

$$
a_{i}=a_{j}=a_{k}
$$

Hence if $R$ is a black region of $D$ with boundary arcs labelled successively $i_{1}, i_{2}, \ldots, i_{r}$ (see Figure 4), then $a_{i_{1}}=a_{i_{2}}=\ldots=a_{i_{r}}$. Equation (8) shows that the $a_{l}$ determined by boundary arcs of black regions sharing a corner are the same. Since any two black regions are connected by a chain of black regions for which successive regions share a corner, it follows that $a_{1}=a_{2}=\ldots=a_{n}$. Hence $\pi(D) \cong \pi_{1}(\Sigma(L)) * \mathbb{Z}$ is abelian. As we noted above, this implies Theorem 8 . 


\section{Surgeries on ALternating KNOtS}

In this section we prove Propositions 9 and 10, These results provide examples, many hyperbolic, of non-L-spaces with left-orderable fundamental groups.

Proof of Proposition 9. Assertion (1) of this proposition is an immediate consequence of [36. Theorem 1.5] and Proposition 4.

Roberts has shown [43] that $S_{1 / q}^{3}(K)$ admits a taut foliation under the hypotheses of assertions (2) and (3) of this proposition. We claim that it is also atoroidal. Menasco [28, Corollary 1] has shown that prime alternating knots are atoroidal. On the other hand, Patton [39] has shown that an alternating knot which admits an essential punctured torus is either a two-bridge knot or a three-tangle Montesinos knot. As the boundary slopes of these types of alternating knots are even integers [16], [15], $S_{1 / q}^{3}(K)$ is atoroidal under the hypotheses of assertions $(2)$ and (3). As $H_{1}\left(S_{1 / q}^{3}(K)\right)=0$, the foliation is co-oriented and the representation $\pi_{1}\left(S_{1 / q}^{3}(K)\right) \rightarrow$ Homeo $_{+}\left(S^{1}\right)$ provided by Thurston's universal circle construction [5] lifts to $\mathrm{Homeo}_{+}\left(S^{1}\right)$. As this representation is injective, [3, Theorem 1.1 (1)] implies that $\pi_{1}\left(S_{1 / q}^{3}(K)\right)$ is left-orderable. This completes the proof.

Now we proceed to the proof of Proposition 10, Our argument is based on a result of Khoi stated on page 795 of [21] and justified through a reference to a MAPLE calculation, though no details are given. Because of the importance of his result to our treatment, we provide a proof of it below. See Proposition 30 .

Let $M$ denote the exterior of the figure eight knot. We know from [3, Example 3.13] that there are a continuous family of representations with non-abelian image

$$
\rho_{s}: \pi_{1}(M) \rightarrow P S L_{2}(\mathbb{R}), s \geq \frac{1+\sqrt{5}}{2}
$$

and a continuous function

$$
g:\left[\frac{1+\sqrt{5}}{2}, \infty\right) \rightarrow[0, \infty)
$$

such that $g(s)=r \in \mathbb{Q}$ if and only if $\rho_{s}\left(\mu^{p} \lambda^{q}\right)= \pm I$ where $r=\frac{p}{q}$ is a reduced fraction. Further, the image of $g$ contains $[0,4)$.

Consider the universal covering homomorphism $\psi: \widetilde{S L_{2}} \rightarrow P S L_{2}(\mathbb{R})$. The kernel $\mathcal{K}$ of $\psi$ is the centre of $\widetilde{S L_{2}}$ and is isomorphic to $\mathbb{Z}$. There is a lift of $\rho_{s}$ to a homomorphism $\tilde{\rho}_{s}: \pi_{1}(M) \rightarrow \widetilde{S L_{2}}$ since the obstruction to its existence is the Euler class $e\left(\rho_{s}\right) \in H^{2}\left(\pi_{1}(M) ; \mathcal{K}\right) \cong H^{2}(M ; \mathbb{Z}) \cong 0$ [11, Section 6.2]. The set of all such lifts is a transitive $H^{1}\left(\pi_{1}(M) ; \mathcal{K}\right)$ set where for $\phi \in H^{1}\left(\pi_{1}(M) ; \mathcal{K}\right)$

$$
\left(\phi \cdot \widetilde{\rho}_{s}\right)(\gamma)=\phi(\gamma) \widetilde{\rho}_{s}(\gamma)
$$

There is an identification $\widetilde{S L_{2}} \cong \Delta \times \mathbb{R}$ where $\Delta=\{z \in \mathbb{C}:|z|<1\}$ in which the following properties hold: 
- the identity is represented by $(0,0)$;

- $\mathcal{K}$ corresponds to $\{(0, k \pi): k \in \mathbb{Z}\}$

- if the image of $(z, \omega)$ in $S L_{2}(\mathbb{R})$ has positive eigenvalues, then there is an even integer $2 j$ such that $|\omega-2 j \pi|<\frac{\pi}{2}$. Further, $(z, \omega)$ is conjugate to an element of the form $(r, 2 j \pi)$ where $r \in(-1,1)$;

- for $r \in(-1,1)$, the centralizer of $(r, 0)$ is contained in $\bigcup_{j \in \mathbb{Z}}(-1,1) \times\{j \pi\}$.

See [21, Section 2], for instance, for the details.

The action of $P S L_{2}(\mathbb{R})$ on the circle induces an inclusion $\widetilde{S L_{2}} \leq \widetilde{\mathrm{Homeo}_{+}}\left(S^{1}\right)=\{f \in$ Homeo $_{+}(\mathbb{R}): f(x+1)=f(x)+1$ for all $\left.x \in \mathbb{R}\right\}$. In this case,

- if $(z, \omega)$ is a commutator, then $-\frac{3 \pi}{2}<\omega<\frac{3 \pi}{2}$ [49, Inequality 4.4 and Proposition $4.8]$

Proposition 30. (Khoi) Let $\tilde{\rho}: \pi_{1}(M) \rightarrow \widetilde{S L_{2}}$ be a homomorphism. Then up to conjugation and replacing $\tilde{\rho}$ by a representation $\tilde{\rho}^{\prime}=\phi \cdot \widetilde{\rho}$ for some $\phi \in H^{1}\left(\pi_{1}(M) ; \mathcal{K}\right)$, we can suppose that $\tilde{\rho}\left(\pi_{1}(\partial M)\right)$ is contained in the 1-parameter subgroup $(-1,1) \times\{0\}$ of $\widetilde{S L_{2}}$.

Proof of Proposition 10. Let $r=\frac{p}{q} \in[0,4)$ and fix $s$ so that $g(s)=r$. Then $\widetilde{\rho}_{s}\left(\mu^{p} \lambda^{q}\right) \in \mathcal{K}=$ $\{(0, k \pi): k \in \mathbb{Z}\}$. On the other hand, by Proposition 30 we may assume $\tilde{\rho}_{s}\left(\pi_{1}(\partial M)\right) \subset$ $(-1,1) \times\{0\}$. Thus $\tilde{\rho}_{s}\left(\mu^{p} \lambda^{q}\right)=(0,0)=1$. It follows that $\tilde{\rho}_{s}$ induces a homomorphism $\pi_{1}\left(S_{r}^{3}(K)\right) \rightarrow \widetilde{S L_{2}}$ with non-abelian image. Since $S_{r}^{3}(K)$ is irreducible for all $r$ [16, Theorem 2(a)] and $\widetilde{S L_{2}}$ is left-orderable [9], [3, Theorem 1.1] implies that $\pi_{1}\left(S_{r}^{3}(K)\right)$ is left-orderable for all $r \in[0,4)$. Since $K$ is amphicheiral, $\pi_{1}\left(S_{r}^{3}(K)\right)$ is left-orderable for $r \in(-4,0]$, so we are done.

The rest of this section is devoted to the proof of Proposition 30. First we develop some background material.

Consider the presentation

$$
\pi_{1}(M)=\left\langle x, y, t: t x t^{-1}=x y x^{2}, t y t^{-1}=x^{-1}\right\rangle
$$

Here $t$ is a meridional class and $\lambda=[x, y]$ a longitudinal class. The reader will verify that $[t, \lambda]=1$. Set $F=\langle x, y\rangle \triangleleft \pi$.

We denote the $S L_{2}(\mathbb{C}), S L_{2}(\mathbb{R})$, and $S U(2)$ character varieties of a group $\Gamma$ by $X_{S L_{2}(\mathbb{C})}(\Gamma)$, $X_{S L_{2}(\mathbb{R})}(\Gamma)$, and $X_{S U(2)}(\Gamma)$ respectively. The character of a representation $\rho$ will be denoted by $\chi_{\rho}$.

There is a homeomorphism [12, Proposition 4.1]

$$
\Psi: X_{S L_{2}(\mathbb{C})}(F) \cong \mathbb{C}^{3}, \chi \mapsto(\chi(x), \chi(y), \chi(x y))
$$


It is known that

$$
\Psi^{-1}\left(\mathbb{R}^{3}\right)=\left\{\chi_{\rho}: \rho \text { has image in } S L_{2}(\mathbb{R}) \text { or } S U(2)\right\}
$$

See [29, Proposition III.1.1].

Lemma 31. The image of the composition of $\Psi$ with the restriction induced $\operatorname{map}_{S_{L_{2}}(\mathbb{C})}(\pi) \rightarrow$ $X_{S L_{2}(\mathbb{C})}(F)$ is $X_{0}=\left\{\left(a, a, \frac{a}{a-1}\right) \in \mathbb{C}^{3}: a \neq 1\right\}$.

Proof. The identity $\operatorname{trace}(A B)+\operatorname{trace}\left(A B^{-1}\right)=\operatorname{trace}(A) \operatorname{trace}(B)$ for $A, B \in S L_{2}(\mathbb{C})$ implies that for each $\chi \in X_{S L_{2}(\mathbb{C})}\left(\pi_{1}(M)\right)$ and $z, w \in \pi_{1}(M)$ we have

$$
\chi(z w)+\chi\left(z w^{-1}\right)=\chi(z) \chi(w)
$$

Given such a $\chi$ set $a=\chi(x)$. The relation $x^{-1}=t y t^{-1}$ implies that $\chi(y)=a$. Next note that $\chi(x y)=\chi\left(t x y t^{-1}\right)=\chi(x y x)=\chi(x y x)+\chi\left(x y x^{-1}\right)-\chi\left(x y x^{-1}\right)=\chi(x y) \chi(x)-\chi(y)=$ $a \chi(x y)-a$. Thus $\chi(x y)=\frac{a}{a-1}$ so the image of the composition of $\Psi$ with the restriction induced map $X_{S L_{2}(\mathbb{C})}(\pi) \rightarrow X_{S L_{2}(\mathbb{C})}(F)$ is contained in $X_{0}$.

Conversely fix $\left(a, a, \frac{a}{a-1}\right) \in X_{0}$ and consider the isomorphism $\theta: F \rightarrow F$ given by $x \mapsto$ $x y x^{2}, y \mapsto x^{-1}$. There is a semisimple representation $\rho_{0}: \pi_{1}(F) \rightarrow S L_{2}(\mathbb{C})$ such that $\Psi\left(\chi_{\rho_{0}}\right)=\left(a, a, \frac{a}{a-1}\right)$. Let $\rho_{1}=\rho_{0} \circ \theta$. It is easy to see that $\rho_{0}$ and $\rho_{1}$ have the same character. Since they are semisimple there is an $A \in S L_{2}(\mathbb{C})$ such that $\rho_{1}=A \rho_{0} A^{-1}$. It is easy to see then that there is a representation $\rho: \pi \rightarrow S L_{2}(\mathbb{C})$ such that $\rho(t)=A$ and $\rho \mid \pi_{1}(F)=\rho_{0}$. Hence $\left(a, a, \frac{a}{a-1}\right)$ lies in the image of image of the composition of $\Psi$ with the restriction induced map $X_{S L_{2}(\mathbb{C})}(\pi) \rightarrow X_{S L_{2}(\mathbb{C})}(F)$, which completes the proof of the lemma.

Let $\kappa: \mathbb{C}^{3} \rightarrow \mathbb{C}$ be given by

$$
\kappa(a, b, c)=a^{2}+b^{2}+c^{2}-a b c-2
$$

Then for $\chi \in X_{S L_{2}(\mathbb{C})}(F)$ and $(\chi(x), \chi(y), \chi(x y))=(a, b, c)$, Identity (9) implies that $\chi([x, y]))=\kappa(a, b, c)$.

Proposition 32. [12, Theorem 4.3] Let $\chi \in X_{S L_{2}(\mathbb{C})}(F)$ and set $(a, b, c)=(\chi(x), \chi(y), \chi(x y))$. Then $\chi \in X_{S U(2)}(F)$ if and only if $(a, b, \kappa(a, b, c)) \in[-2,2]^{3}$.

A straightforward calculation shows that for $a \in \mathbb{R} \backslash\{1\}$,

- $\kappa\left(a, a, \frac{a}{a-1}\right)=(a-1)^{2}+(a-1)-2+(a-1)^{-1}+(a-1)^{-2} \geq-2$, and

- $\left(a, a, \kappa\left(a, a, \frac{a}{a-1}\right)\right) \in[-2,2]^{3}$ if and only if $-\left(\frac{\sqrt{5}+1}{2}\right) \leq a \leq \frac{\sqrt{5}-1}{2}$ or $a=2$.

Lemma 33. The image of the composition of $\Psi$ with the restriction map $X_{S L_{2}(\mathbb{R})}\left(\pi_{1}(M)\right) \rightarrow$ $X_{S L_{2}(\mathbb{R})}(F)$ is $Y_{0}=\left\{\left(a, a, \frac{a}{a-1}\right): a \in \mathbb{R} \backslash\{1\}, \kappa\left(a, a, \frac{a}{a-1}\right) \geq 2\right\}$. In particular, if $\chi_{\rho} \in$ $X_{S L_{2}(\mathbb{R})}\left(\pi_{1}(M)\right)$, the eigenvalues of $\rho(\lambda)$ are positive reals. 
Proof. Fix $\chi_{\rho} \in X_{S L_{2}(\mathbb{R})}\left(\pi_{1}(M)\right)$. If $\rho$ is reducible, then $\chi\left(\pi_{1}(F)\right)=\{2\}$ since $\pi_{1}(F)$ is contained in the commutator subgroup of $\pi_{1}(M)$. Thus $\Psi\left(\chi_{\rho}\right)=(2,2,2) \in Y_{0}$. Suppose then that $\chi_{\rho}$ is irreducible. The image of $\rho$ leaves a geodesic plane $P$ in $\mathbb{H}^{3}$ invariant so it cannot conjugate into $S U(2)$; otherwise it would fix a point of $P$ and therefore be conjugate into $S O(2)$ contrary to the irreduciblity of $\rho$. Thus $\Psi\left(\chi_{\rho}\right) \notin X_{S U(2)}\left(\pi_{1}(M)\right)$. It follows from Proposition 32 that $\Psi\left(\chi_{\rho}\right) \in\left\{\left(a, a, \frac{a}{a-1}\right): a \in \mathbb{R} \backslash\{1\}, \kappa\left(a, a, \frac{a}{a-1}\right) \notin[-2,2]\right\}$. Since $\kappa\left(a, a, \frac{a}{a-1}\right) \geq-2$ for all $a \in \mathbb{R} \backslash\{1\}, \Psi\left(\chi_{\rho}\right) \in Y_{0}$.

Finally observe that if $\chi_{\rho} \in X_{S L_{2}(\mathbb{R})}\left(\pi_{1}(M)\right)$ and $\rho(\lambda)$ has eigenvalues $\zeta, \zeta^{-1} \in \mathbb{C}^{*}$, then $\zeta+\zeta^{-1}=\operatorname{trace}(\rho(\lambda))=\kappa\left(a, a, \frac{a}{a-1}\right) \geq 2$. Thus $\zeta$ is a positive real number.

Proof of Proposition 30. The properties of $\widetilde{S L_{2}}=\Delta \times \mathbb{R}$ listed just before the statement of Proposition 30 will be used without direct reference in the proof.

Since $\lambda$ is a commutator, if $\tilde{\rho}(\lambda)=(z, \omega)$ then $-\frac{3 \pi}{2}<\omega<\frac{3 \pi}{2}$. On the other hand, since the eigenvalues of $\psi(\tilde{\rho}(\lambda))$ are positive there is an even integer $2 k$ such that $|\omega-2 k \pi|<\frac{\pi}{2}$. Hence $-\frac{\pi}{2}<\omega<\frac{\pi}{2}$ and therefore $\tilde{\rho}(\lambda)$ is conjugate into the subgroup $(-1,1) \times\{0\}$ of $\widetilde{S L_{2}}$. Without loss of generality we assume $\tilde{\rho}(\lambda) \in(-1,1) \times\{0\}$. Since $\tilde{\rho}(t)$ commutes with $\tilde{\rho}(\lambda)$, there is an integer $j$ such that $\tilde{\rho}(t) \in(-1,1) \times\{j \pi\}$. Fix $\phi_{0} \in H^{1}(M ; \mathcal{K})=\operatorname{Hom}\left(\pi_{1}(M), \mathcal{K}\right)$ such that $\phi_{0}(t)=(0,-j \pi)$ and set $\tilde{\rho}^{\prime}=\phi \cdot \widetilde{\rho}$. From the multiplication on $\widetilde{S L_{2}}$ (cf. [21, page $764])$ we see that $\tilde{\rho}^{\prime}(t) \subset(-1,1) \times\{0\}$. Then $\tilde{\rho}^{\prime}\left(\pi_{1}(\partial M)\right) \subset(-1,1) \times\{0\}$, which completes the proof.

\section{Left-orderability and Representations with Values in $\operatorname{HomeO}_{+}\left(S^{1}\right)$}

In this section we prove Theorem [12, Corollary 13 and Corollary 14 .

Proof of Theorem 12. Let $K$ be a prime knot in the 3-sphere and suppose that $\pi_{1}(\Sigma(K))$ is not left-orderable. Let $M_{K}$ denote the exterior of $K$ and fix a homomorphism $\rho: \pi_{1}\left(M_{K}\right) \rightarrow$ Homeo $_{+}\left(S^{1}\right)$ such that $\rho\left(\mu^{2}\right)=1$ for each meridional class $\mu$ in $\pi_{1}\left(M_{K}\right)$. We will show that the image of $\rho$ is either trivial or isomorphic to $\mathbb{Z} / 2$.

Let $p: \widetilde{M}_{K} \rightarrow M_{K}$ be the 2 -fold cover determined by the epimorphism $\pi_{1}\left(M_{K}\right) \rightarrow \mathbb{Z} / 2$. Then $\Sigma(K)$ is obtained by filling the boundary component of $\widetilde{M}_{K}$ along the inverse image of a meridional curve of $M_{K}$.

The Euler class $e(\rho)$ of $\rho\left(\left[11\right.\right.$, Section 6.2]) is contained in $H^{2}\left(M_{K} ; \mathbb{Z}\right) \cong 0$, and so is zero. Hence if $\widetilde{\rho}=\rho \mid \pi_{1}\left(\widetilde{M}_{K}\right)$, then $e(\widetilde{\rho})=p^{*}(e(\rho))=0$. Our assumptions imply that $\widetilde{\rho}$ induces a homomorphism $\psi: \pi_{1}(\Sigma(K)) \rightarrow$ Homeo $_{+}\left(S^{1}\right)$ such that if $i: \widetilde{M}_{K} \rightarrow \Sigma(K)$ is the inclusion, $e(\widetilde{\rho})=i^{*}(e(\psi))$.

Let $\widehat{K}=p^{-1}(K) \subset \Sigma(K)$ and let $N(\widehat{K})$ denote a closed tubular neighbourhood of $\widehat{K}$ in $\Sigma(K)$. Note that $H_{1}\left(\widetilde{M}_{K} ; \mathbb{Z}\right) \cong H_{1}(\Sigma(K) ; \mathbb{Z}) \oplus H_{2}\left(\Sigma(K), \widetilde{M}_{K} ; \mathbb{Z}\right) \cong H_{1}(\Sigma(K) ; \mathbb{Z}) \oplus \mathbb{Z}$ where 
the $\mathbb{Z}$ factor is generated by the boundary of a meridian disk of $N(\widehat{K})$. It follows that the connecting homomorphism $H^{1}\left(\widetilde{M}_{K} ; \mathbb{Z}\right)=\operatorname{Hom}\left(H_{1}\left(\widetilde{M}_{K} ; \mathbb{Z}\right), \mathbb{Z}\right) \rightarrow H^{2}\left(\Sigma(K), \widetilde{M}_{K} ; \mathbb{Z}\right)=$ $\operatorname{Hom}\left(H_{2}(N(\widehat{K}), \partial N(\widehat{K}) ; \mathbb{Z}) ; \mathbb{Z}\right)$ is surjective. Thus $i^{*}: H^{2}(\Sigma(K) ; \mathbb{Z}) \rightarrow H^{2}\left(\widetilde{M}_{K} ; \mathbb{Z}\right)$ is injective. Then as $i^{*}(e(\psi))=e(\widetilde{\rho})=0, e(\psi)=0$. In particular, $\psi$ lifts to a homomorphism $\widetilde{\psi}: \pi_{1}(\Sigma(K)) \rightarrow{\widetilde{\text { Homeo }_{+}}}_{+}\left(S^{1}\right) \leq$ Homeo $_{+}(\mathbb{R})$ [11, Section 6.2]. Since $K$ is prime, $\Sigma(K)$ is irreducible. Further, Homeo $+(\mathbb{R})$ is left-orderable [30, Theorem 7.1.2], and therefore as $\pi_{1}(\Sigma(K))$ is not left-orderable, $\widetilde{\psi}$ is the trivial homomorphism [3, Theorem 1.1]. The same conclusion then holds for $\widetilde{\rho}$ and hence the image of $\rho$ is a cyclic group of order dividing 2 .

Proof of Corollary 13, Let $K$ be an alternating knot and $\rho: \pi_{1}\left(S^{3} \backslash K\right) \rightarrow \operatorname{Homeo}_{+}\left(S^{1}\right)$ a homomorphism such that $\rho\left(\mu^{2}\right)=1$ for each meridional class $\mu$ in $\pi_{1}\left(S^{3} \backslash K\right)$. Corollary 13 clearly holds when $K$ is trivial, so suppose it isn't and let $K_{1}, K_{2}, \ldots, K_{n}$ be its prime factors. Each $K_{i}$ is alternating and

$$
\pi_{1}\left(S^{3} \backslash K\right) \cong \pi_{1}\left(S^{3} \backslash K_{1}\right) *_{\mu_{1}=\mu_{2}} \pi_{1}\left(S^{3} \backslash K_{2}\right) *_{\mu_{2}^{\prime}=\mu_{3}} \ldots *_{\mu_{n-1}^{\prime}=\mu_{n}} \pi_{1}\left(S^{3} \backslash K_{n}\right)
$$

where $\mu_{i}, \mu_{i}^{\prime}$ are meridional classes of $K_{i}$. Further, a meridional class of $K_{i}$ is a meridional class of $K$. Hence Theorem 12 implies that for each $i, \rho\left(\pi_{1}\left(S^{3} \backslash K_{i}\right)\right)$ is a subgroup of $\mathbb{Z} / 2$. Then $\rho \mid \pi_{1}\left(S^{3} \backslash K_{i}\right)$ factors through $H_{1}\left(S^{3} \backslash K_{i}\right)$ and therefore $\rho\left(\pi_{1}\left(S^{3} \backslash K_{i}\right)\right)$ is generated by $\rho\left(\mu_{i}\right)$ and $\rho\left(\mu_{i}\right)=\rho\left(\mu_{i}^{\prime}\right)$ for $2 \leq i \leq n-1$. Given our presentation for $\pi_{1}\left(S^{3} \backslash K\right)$, Corollary 13 is a straightforward consequence of these observations.

Proof of Corollary 14. Finally, consider the hypotheses of Corollary 14 and let $M_{K}$ be the exterior of $K$. If the trace field of $\pi_{1}\left(\mathcal{O}_{K}(2)\right)$ has a real embedding, then it determines an irreducible representation of $\pi_{1}\left(M_{K}\right)$ which conjugates into $P S L_{2}(\mathbb{R})$ or $P S U(2)$ [27, Definition 7.2.1]. The former is ruled out by Corollary [13, Thus Corollary [14 holds.

\section{REFERENCES}

[1] J. Berge, Some knots with surgeries yielding lens spaces, unpublished manuscript.

[2] S. Boyer, Dehn surgery on knots, In Handbook of Geometric Topology, North-Holland, Amsterdam, 2002, 165-218.

[3] S. Boyer, D. Rolfsen, and B. Wiest, Orderable 3-manifold groups, Ann. Inst. Fourier 55 (2005), 243-288.

[4] R. Burns and V. Hale, A note on group rings of certain torsion-free groups, Can. Math. Bull. 15 (1972), 441-445.

[5] D. Calegari and N. Dunfield, Laminations and groups of homeomorphisms of the circle, Inv. Math. 152 (2003), 149-2004.

[6] A. Clay, T. Lidman and L. Watson, Graph manifolds, left-orderability and amalgamation, arxiv:1106.0486.

[7] A. Clay and L. Watson, Left-orderable fundamental groups and Dehn surgery, arXiv:1009.4176.

[8] - On cabled knots, Dehn surgery, and left-orderable fundamental groups, arXiv:1103.2358.

[9] P. Conrad, Right-ordered groups, Michigan Math. J. 6 (1959), 267-275.

[10] M. Culler, Lifting representations to covering groups, Adv. in Math. 59 (1986), 64-70.

[11] E. Ghys, Groups acting on the circle, L'Enseignement Math. 22 (2001), 329-407.

[12] W. Goldman, Topological components of spaces of representations, Inv. Math. 93 (1998), 557-607. 
[13] F. Gonzàlez-Acuna and J.-M. Montesinos-Amilibia, On the character variety of group representations in $S L(2, C)$ and PSL(2,C), Math. Z. 214 (1993), 627-652.

[14] J. Greene, Alternating links and left-orderability, preprint.

[15] A. Hatcher and U. Oertel, Boundary slopes for Montesinos knots, Topology 28 (1989), 453-480.

[16] A. Hatcher and W. Thurston, Incompressible surfaces in 2-bridge knot complements, Inv. Math. 79 (1985), 225-246.

[17] W. Heil, Elementary surgery on Seifert fiber spaces, Yokohama Math. J. 47 (1974), 135-139.

[18] J. Howie and H. Short, The band-sum problem, J. London Math. Soc. (2), 31 (1985), 571-576.

[19] T. Ito, Non-left-orderable double branched coverings, preprint, arXiv:1106.1499.

[20] A. Juhász, Holomorphic discs and sutured manifolds, Algebr. Geom. Topol. 6 (2006), 1429-1457.

[21] V. Th. Khoi, A cut-and-paste method for computing the Seifert volumes, Math. Ann. 326 (2003), 759801.

[22] A. Levine, Knot doubling operators and bordered Heegaard Floer homology, preprint, arXiv:1008.3349.

[23] A. Levine and S. Lewallen, private communication.

[24] R. Lipshitz, P. Ozsváth, and D. Thurston, Bimodules in bordered Heegaard Floer homology, preprint, arXiv:1003.0598.

[25] - Bordered Heegaard Floer homology, preprint, arXiv:0810.0687.

[26] P. Lisca and A. Stipsicz, Ozsváth-Szabó invariants and tight contact 3-manifolds. III, J. Symplectic Geom. 5 (2007), 357-384.

[27] C. MacLachlan and A. Reid, The Arithmetic of Hyperbolic 3-Manifolds, GTM 219, SpringerVerlag New York Inc., 2003.

[28] W. Menasco, Closed incompressible surfaces in alternating knot and link complements, Topology 23 (1984), 37-44

[29] J. Morgan and P. Shalen, Valuations, trees, and degenerations of hyperbolic structures, I, Annals Math 23 (1984), 401-476.

[30] R. Mura and A. Rhemtulla, Orderable Groups, Dekker Lecture Notes in Pure and Appl. Math. 27, 1977.

[31] P. Ozsváth and Z. Szabó. Holomorphic disks and genus bounds, Geom. Topol. 8 (2004), 311-334 (electronic).

[32] - Holomorphic disks and three-manifold invariants: properties and applications, Ann. of Math. 159 (2004), 1159-1245.

[33] - Holomorphic disks and topological invariants for closed three-manifolds, Ann. of Math. 159 (2004), 1027-1158.

[34] - Holomorphic disks and knot invariants, Adv. Math. 186 (2004), 58-116.

[35] - On Heegaard diagrams and holomorphic disks, In European Congress of Mathematics, Eur. Math. Soc., Zürich, 2005, 769-781.

[36] —, On knot Floer homology and lens space surgeries, Topology 44 (2005), 1281-1300.

[37] - On the Heegaard Floer homology of branched double-covers, Adv. Math. 194 (2005), 1-33.

[38] - Lectures on Heegaard Floer homology, In Floer homology, gauge theory, and lowdimensional topology, volume 5 of Clay Math. Proc., Amer. Math. Soc., Providence, RI, 2006, pages 29-70.

[39] R. Patton, Incompressible punctured tori in the complements of alternating knots, Math. Ann. 301 (1995), 1-22.

[40] T. Peters, On L-spaces and non left-orderable 3-manifold groups, arxiv:0903.4495.

[41] J. H. Przytycki, 3-coloring and other elementary invariants of knots, Knot Theory, Banach Center Publications 42, Institute of Mathematics, Polish Academy of Sciences, Warsaw 1998, 275-295.

[42] J. Rasmussen, Floer homology and knot complements, PhD thesis, Harvard University, 2003.

[43] R. Roberts, Constructing taut foliations, Comm. Math. Helv. 70 (1995), 516-545.

[44] P. Scott, The geometries of 3-manifolds, Bull. London Math. Soc. 15 (1983), 401-487.

[45] A. A. Vinogradov, On the free product of ordered groups, Mat. Sbornik N.S. 25 (1949), 163-168.

[46] M. Wada, Group invariants of links, Topology 31 (1992), 399-406.

[47] L. Watson, Surgery obstructions from Khovanov homology, arxiv:0807.1341. 
[48] - Involutions on 3-manifolds and Khovanov homology, $\mathrm{PhD}$ thesis, Université du Québec à Montréal, 2009.

[49] J. W. Wood, Bundles with totally disconnected structure group, Comment. Math. Helv. 46 (1971), $257-273$.

Département de Mathématiques, Université du Québec à Montréal, 201 avenue du PrésidentKennedy, Montréal, QC H2X 3Y7.

E-mail address: boyer.steven@uqam.ca

$U R L:$ http://www.cirget.uqam.ca/boyer/boyer.html

Department of Mathematics, University of Texas at Austin, 1 University Station, Austin, TX 78712 .

E-mail address: gordon@math.utexas.edu

URL: http://www.ma.utexas.edu/text/webpages/gordon.html

Department of Mathematics, UCla, 520 Portola Plaza, los Angeles, CA 90095.

E-mail address: lwatson@math.ucla.edu

$U R L:$ http: //www.math.ucla.edu/ Iwatson 